\title{
Under-Track CFD-Based Shape Optimization for a Low-Boom Demonstrator Concept
}

\author{
Mathias Wintzer* \\ Analytical Mechanics Associates, Inc., Hampton, VA 23666 \\ Irian Ordaz ${ }^{\dagger}$ \\ NASA Langley Research Center, Hampton, VA 23681 \\ James W. Fenbert ${ }^{\ddagger}$ \\ Analytical Mechanics Associates, Inc., Hampton, VA 23666
}

\begin{abstract}
The detailed outer mold line shaping of a Mach 1.6, demonstrator-sized low-boom concept is presented. Cruise trim is incorporated a priori as part of the shaping objective, using an equivalent-area-based approach. Design work is performed using a gradient-driven optimization framework that incorporates a three-dimensional, nonlinear flow solver, a parametric geometry modeler, and sensitivities derived using the adjoint method. The shaping effort is focused on reducing the under-track sonic boom level using an inversedesign approach, while simultaneously satisfying the trim requirement. Conceptual-level geometric constraints are incorporated in the optimization process, including the internal layout of fuel tanks, landing gear, engine, and crew station. Details of the model parameterization and design process are documented for both flow-through and powered states, and the performance of these optimized vehicles presented in terms of inviscid $L / D$, trim state, pressures in the nearfield and at the ground, and predicted sonic boom loudness.
\end{abstract}

\section{Introduction}

$\mathrm{T}$ HE field of sonic boom minimization has advanced steadily since the pioneering work of Seebas, George and Darden. ${ }^{1,2,3}$ The application of potential theory methods to the conceptual design of low-boom aircraft has been demonstrated by Farhat et al. ${ }^{4}$ while similar work additionally considered pressure signals sampled in the near-field of the CFD domain as part of an integrated conceptual design process. ${ }^{5}$ Approaches using gradient-based optimization with non-linear methods were described by Alonso et al. ${ }^{6}$ and the subsequent application of these methods to the development of low-boom supersonic business jets was demonstrated by Choi et al. ${ }^{7}$ More recently, the application of adjoint-based adaptive mesh refinement for increased simulation accuracy has been presented for both tetrahedral ${ }^{8,9}$ as well as Cartesian meshes, ${ }^{10}$ with the latter being validated against flight test data. ${ }^{11}$ In the context of low-boom shape optimization, the use of adjoint-derived sensitivities has been demonstrated using both continuous ${ }^{12}$ and discrete ${ }^{13,14}$ adjoint methods. The effectiveness of these approaches has encouraged their application to a wide range of conceptual design studies. ${ }^{15,16,17,18}$ Propulsion integration has also seen dedicated, extensive study, with methodologies presented for high performance inlet ${ }^{19,20}$ and nozzle ${ }^{21,22}$ design. The synthesis of these recent advances is a topic of active research, and seeks to enable the development of vehicles that, at least conceptually and in the CFD domain, achieve the low sonic boom levels that may enable lifting of the ban on overland supersonic flight.

In the experimental domain, the shaped sonic boom demonstrator ${ }^{23}$ (SSBD) was the first to apply many of these technologies to a flight test demonstrator, where the theoretical basis relating vehicle shape to the sonic boom signature at the ground was proven in flight test. Preparation for a follow-on experiment is

*Engineer, Analytical Mechanics Associates, Inc., 21 Enterprise Parkway, Suite 300. Senior Member AIAA.

${ }^{\dagger}$ Aerospace Engineer, Aeronautics Systems Analysis Branch, Mail Stop 442. Member AIAA.

‡Engineer, Analytical Mechanics Associates, Inc., 21 Enterprise Parkway, Suite 300. 
presently underway that aims to determine public acceptance of a fully shaped low sonic boom signature using a flight test article. To that end, current efforts are focused on the design of a small, lightweight vehicle by NASA and industry partners.

This paper presents ongoing work aimed at developing a reference design that satisfies the mission requirements defined for this follow-on vehicle, illustrated in Fig. 1. In particular, this work documents the CFD-based, under-track shape optimization of a low-boom vehicle that achieves trim in cruise; a closely related development effort is focused on off-track shaping. ${ }^{24}$ In this study, the trim requirement is enforced as part of the shaping process using a novel approach presented in recent work. ${ }^{25}$ To ensure a realistic vehicle outer mold line (OML), the integration of key internal components such as the engine, landing gear, and crew station is also considered. The shaping process follows two distinct phases, with the propulsion system initially modeled as a flow-through element. The subsequent refinement phase describes the re-tailoring of the vehicle required once active power boundary conditions are included, and modeling approaches and experience gained from a prior low-boom propulsion integration study ${ }^{26}$ are applied here. In practice, the

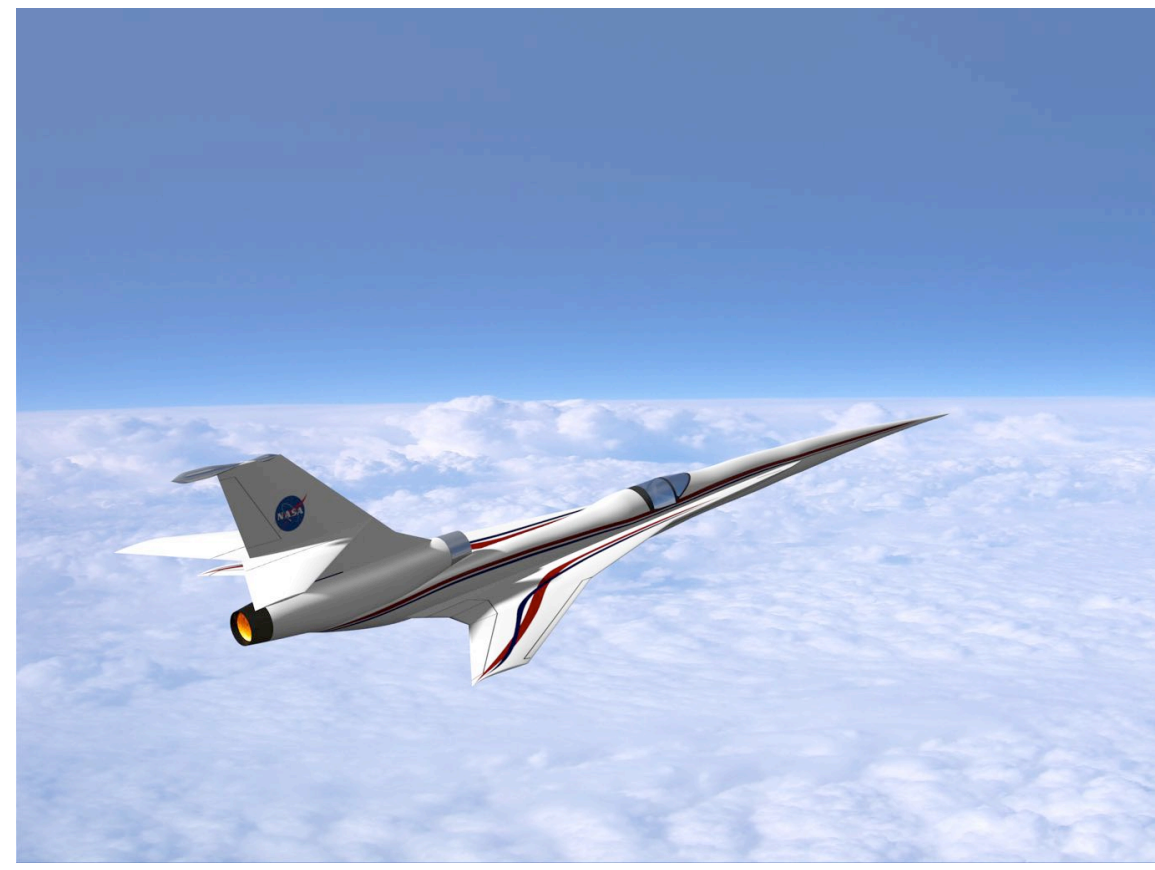

Figure 1. Illustration of the low-boom demonstrator concept

model parameterization evolves as knowledge of the design space is gained; in this study, as in Ref. 26, changes are introduced manually, between design cycles. Recent work by Anderson and Aftosmis ${ }^{27}$ describes an approach seeking to automate parameter refinement by using surface adjoint indicators to deterministically re-locate or enrich the available set of design variables. In addition to improving the efficiency of the optimization process, the approach promises to remove the need for the designer-in-the-loop.

The concept selected for further development in this work is the "low boom feasible" $108 \mathrm{ft}$ demonstrator described in Ref. 25, notionally illustrated in Fig. 2. The cruise design point is Mach 1.6 at an altitude of $51,700 \mathrm{ft}$. The $C_{L}$ at this condition is 0.065 , corresponding to a $21,000 \mathrm{lb}$ cruise weight.

\section{Design Approach}

In Ref. 25, a mixed-fidelity technique is used to generate a low-boom target profile that, in addition to incorporating lift and volumetric constraints, accounts for a cruise trim requirement. Evaluation of the equivalent area $\left(A_{e}\right)$ distribution using an off-body, nonlinear analysis enables development of a configuration layout with trim performance that persists in the CFD domain. In this context, the term feasible implies a baseline configuration with $A_{e}$ sufficiently close to the desired target that, following detailed OML shaping, will successfully recover the target sonic boom loudness level.

In this work, an adjoint-based, inverse design approach is used to drive the OML shape such that the pressure distribution sampled in the nearfield matches that of the specified target. Surface geometry is 


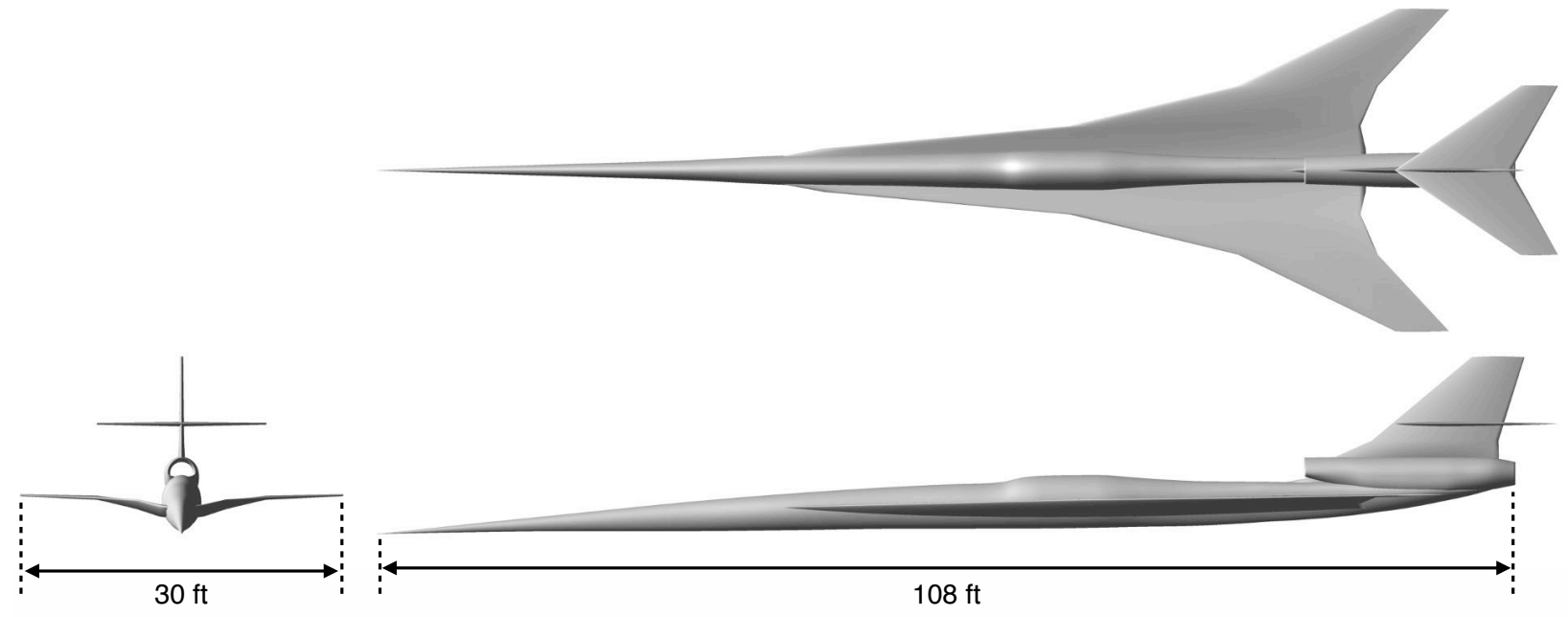

Figure 2. Three-view diagram of the baseline concept

generated using a parametric modeler, with bound constraints used to prevent intrusion of the OML into the cockpit station, landing gear bays, and engine. Nonlinear constraints, specified as described in Ref. 26, are used to ensure airfoil sections remain physically realizable. Atmospheric propagation of nearfield pressure distributions sampled in the CFD domain is performed using sBOOM, ${ }^{28}$ and uses the ANSI S1.26-1995 standard atmosphere. ${ }^{29}$ Estimation of the perceived level of loudness ${ }^{30}$ (PLdB) from the resultant ground pressure signal uses the approach of Shepherd and Sullivan. ${ }^{31}$

\section{II.A. Adjoint-Driven CFD Shape Optimization}

This study uses the Cart3D ${ }^{32}$ CFD design framework. It employs a multilevel, embedded boundary Cartesian mesh, and provides a robust and efficient parallel multigrid solver for the three-dimensional Euler

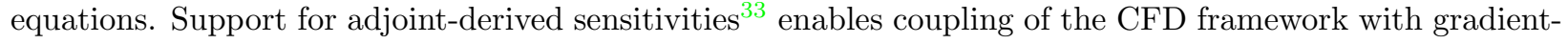
based optimization systems. The Cart3D adjoint capability can also be invoked for mesh adaptation, where adjoint-derived cell-wise error estimates are used to iteratively refine the mesh, minimizing the discretization error in a specified objective functional. Grid stretching and alignment techniques to improve mesh efficiency, along with the validation of this adaptive refinement approach in supersonic flow, are described in Ref. 11.

The SNOPT $^{34}$ constrained optimization system is used to drive the shaping process. SNOPT uses a Sequential Quadratic Programming (SQP) algorithm, solving a Quadratic Programming (QP) subproblem at each major iteration in order to determine the next search direction. Hessian updates are performed using the Broyden-Fletcher-Goldfarb-Shanno (BFGS) formula. The objective function has the form

$$
\mathcal{J}=\int\left(\frac{p-p_{t}}{p_{\infty}}\right)^{2} d s
$$

where $p$ is pressure, $s$ the distance along the pressure sensor, and the subscript $(t)$ denotes a target value. It is noted that consistent with the trim approach of Ref. 25, no explicit pitching moment constraint is required. Satisfaction of the trim constraint is implicit in the provided target, which considers both the base configuration layout and $A_{e}$ in its formulation. The lift requirement is similarly satisfied, as demonstrated in Ref. 26.

Mesh construction is initially performed using an automated, geometry-based approach, ${ }^{35}$ with prespecified regions of refinement used to propagate the vehicle shock system to the near-field sampling region. The location and extent of these high refinement regions was guided by observation of an adaptively refined mesh structure developed for the baseline geometry. For realistic configurations where the vehicle shock system can become more complex as the design evolves, a mesh with fixed structure may not always capture the flow field with sufficient accuracy, giving rise to discrepancies between the fixed- and adapted-mesh solutions. In cases where coarse, pre-specified meshes are used to increase design cycle throughput, a final, apparently successful design can be found to perform substantially worse when scrutinized by an adaptively refined mesh. In this work, adaptive mesh refinement is invoked during the final stages of design, using 
an approach demonstrated by Nemec and Aftosmis. ${ }^{36}$ While potentially resource-intensive, by tailoring the volume grid to each instance of the geometry, flow features are more accurately captured, discretization error levels are consistent from grid to grid, and the final design maintains its performance under high-resolution analysis.

\section{II.B. Geometry Modeling}

The JAGUAR parametric geometry modeler is used for this study, and is described in detail in Ref. 18. It provides sufficient flexibility to model full aircraft configurations, and offers native computation of surface sensitivities used during gradient-based shape optimization. Sensitivities associated with geometric features are also computed, allowing for the imposition of constraints on properties such as area, volume, and thickness in a way that can be efficiently handled by a gradient-based optimizer. As implemented, the modeler retains much of the flexibility afforded by CAD-based geometry generation systems while offering significant time savings, especially as the number of design parameters is increased.

\section{II.C. Model Parameterization}

The initial parametric model generated for this study, defined using roughly 140 shape design variables, is shown in Fig. 3. As the optimization proceeded, enrichment of this parameterization was required to enable more fine-grained control over the OML shape; notable additions will be discussed in $\S$ III.

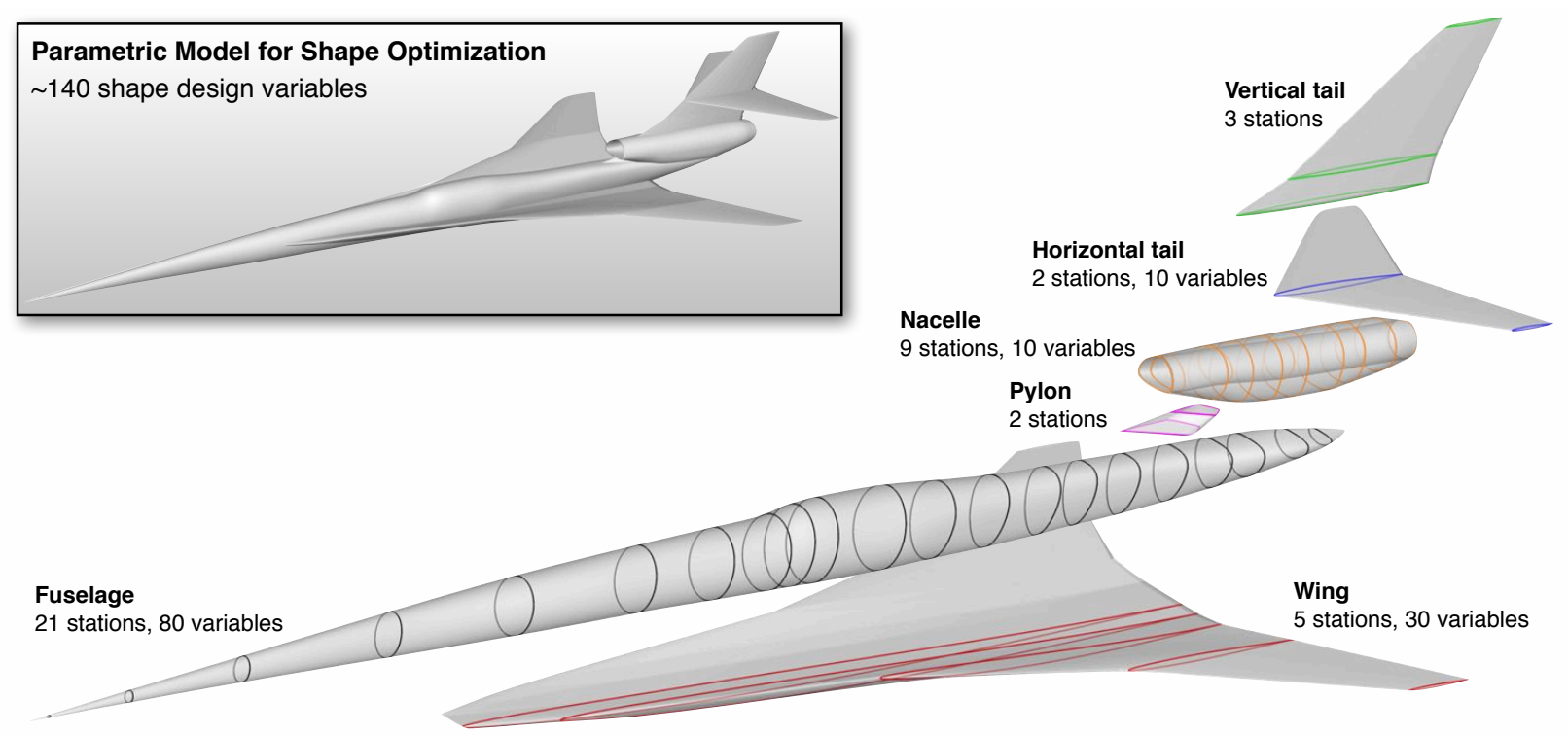

Figure 3. Parametric geometry used during shape optimization

The wing component was defined using a five-section linear lofting. The two innermost sections were defined using a 4th-order Kulfan ${ }^{37}$ parameterization, while the outer three sections used a NACA 65-series thickness profile with a parabolic camber distribution; both the camber crest location and amount were allowed to vary. The incidence angle of the outer three sections was allowed to vary. To ensure adequate structural depth, section thickness-to-chord $(t / c)$ ratios were constrained on the Kulfan-defined airfoils to be no less than $1.4 \%$ and $2.1 \%$ for the root and adjacent sections, respectively. The outer three sections, from inboard to tip section, had fixed $t / c$ of $2.46 \%, 3 \%$, and $3.54 \%$, respectively.

The forward section of the fuselage component was defined using a mixture of circular and elliptic sections, with the cockpit station described using two elliptic sections constrained to be at least $5 \mathrm{ft}$ high and $3.75 \mathrm{ft}$ wide. Aft of the cockpit, split superellipse sections (see Ref. 26) were used to enable the more complex cross-section shapes needed to integrate the nacelle component. All section parameters were activated during shape optimization; radius for circular sections, height and width for elliptic sections, and width, upper height, lower height, upper exponent, and lower exponent for superelliptic sections. Fuselage nose droop was allowed to vary, and effected by shearing of the lofted sections along a parabolic arc. The fuselage was lofted using a monotone cubic Hermite spline. 
The outer surface of the nacelle component was defined using split superelliptic sections up to the nozzle region, which was defined using circular sections. Along with the inlet face, the nozzle section parameters were specified by the engine model at the cruise power state and held fixed. Having to accommodate the inlet duct and propulsion system, the nacelle OML shape was heavily constrained; typically, only section widths were allowed to vary. The internal flow-through duct was defined with superelliptic sections up to the nominal fan face location, after which circular sections were specified. Duct geometry was held fixed.

The horizontal tail used an identical section parameterization as for the outer wing panel, with a $4 \%$ thick root and $5 \%$ thick tip section. The tail was treated as full-flying, and its incidence allowed to vary. The tip section incidence was also allowed to vary independently.

The diverter and vertical tail geometry were fixed throughout the optimization. The vertical tail was constructed using biconvex sections that were $4 \%$ thick at the root, and $6 \%$ thick at the tip. The diverter presented a simple wedge profile to the flow.

\section{II.D. Geometric Constraints due to Internal Systems}

Bounds on the geometry parameters were specified to ensure that major internal systems were always accommodated by the OML. These components were modeled in Open Vehicle Sketch $\mathrm{Pad}^{38}$ using publicly available data.

The cockpit, shown in Fig. 4, is derived from the Northrop T-38 aft crew station. The vehicle incorporates an integral, aft-mounted General Electric F404-GE-402 afterburning turbofan; the structural support frames and airframe-mounted accessory drive (AMAD) as installed in the Boeing F/A-18 are retained. The landing gear from a Lockheed-Martin F-16 is used, with careful attention paid to the stowed configuration of the main gear to better capture the size and shape of its bounding volume.

While not incorporated as an active constraint, the fuel tank geometry was assessed periodically from the surface model to determine volume and center of gravity (CG). The tank layout was modified as needed to ensure sufficient fuel capacity existed to complete the mission, and that the fuel CG could be placed to satisfy trim.

The internal placement of these components is illustrated in Fig. 5, with fuel tank size and position as they would be within the final optimized

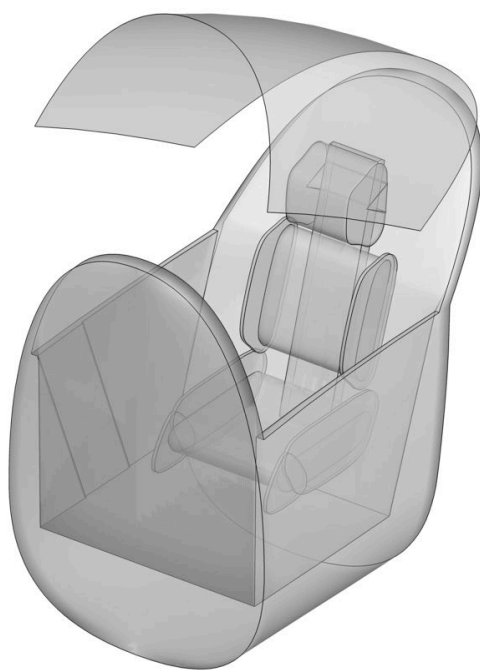

Figure 4. Crew station model OML shape.

\section{Low Boom Design Process}

The shape optimization methodology follows closely from Ref. 26. The nearfield pressure sensor was initially located at an offset distance $h / L$ of two body-lengths, where $h$ is the vertical distance from vehicle nose to sensor, and $L$ the effective vehicle length. This target location was selected to increase cycle throughput, with the expectation that at this distance, shock system coalescence was likely insufficient for the assumptions made by propagation theory to hold. To account for this, initial $h / L=2$ shaping was followed by a refinement phase with the target set at a more appropriate offset distance.

The volume grid used when optimizing to the $h / L=2$ target contained approximately 14 million cells. The nearfield pressure and propagated ground signatures for the target and baseline design are shown in Fig. 6. In this initial state, the ground signature loudness was predicted to be $81.5 \mathrm{PLdB}$ for the baseline design; the target signature loudness is $69.6 \mathrm{PLdB}$. Trim state is monitored through the $x$-location of the center of pressure, $x_{\mathrm{cp}}$. For the baseline design, $x_{\mathrm{cp}}=82.0 \mathrm{ft}$. The configuration can accommodate some forward motion of the $x_{\mathrm{cp}}$ relative to this value via fuel-pumping; hence values slightly less than the baseline value were still considered to satisfy trim. At the design point, the angle of attack $\alpha=3.375^{\circ}$, at which total lift coefficient $C_{L}=0.066$, and inviscid lift-to-drag ratio $\mathrm{L} / \mathrm{D}=7.1$.

Figure 7 shows contours of the density adjoint for the functional given in Eq. 1 plotted on the surface model and symmetry plane. Visualizations of this form can be instructive. By relocating or introducing design parameters to coincide with these regions of high sensitivity, the optimizer is given authority where shape changes have the greatest potential for affecting the objective functional, subject to constraints. 


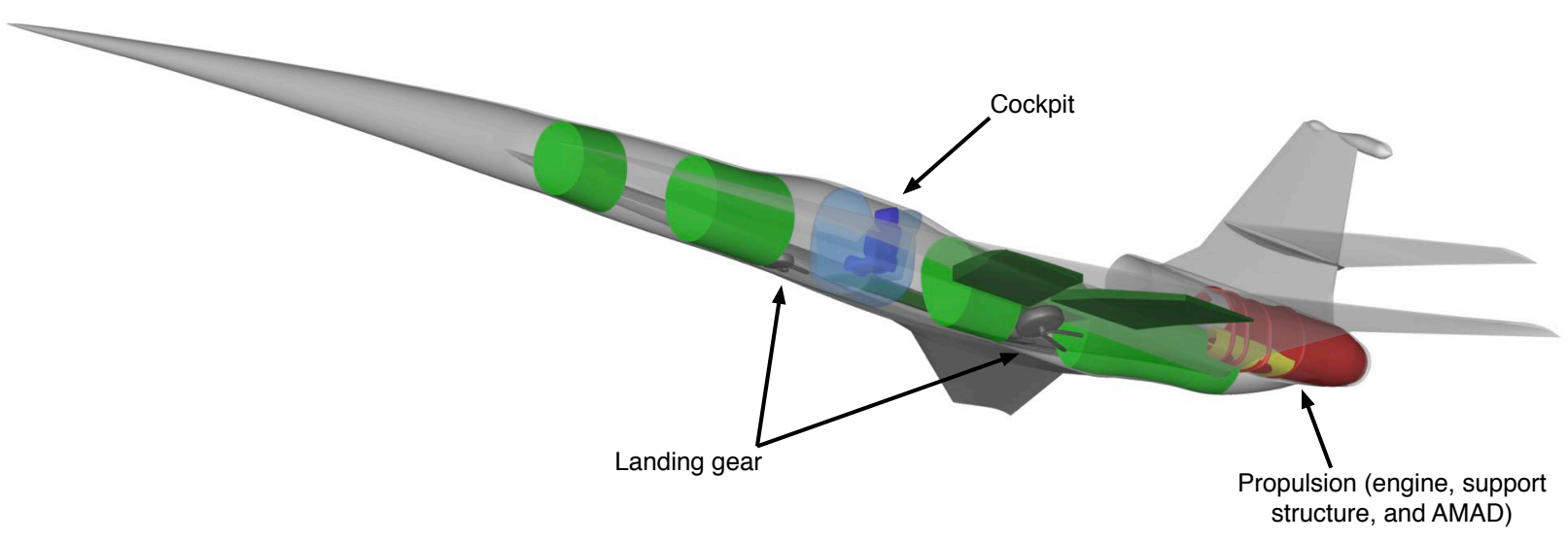

(a) Cockpit, landing gear and propulsion system positioning within vehicle

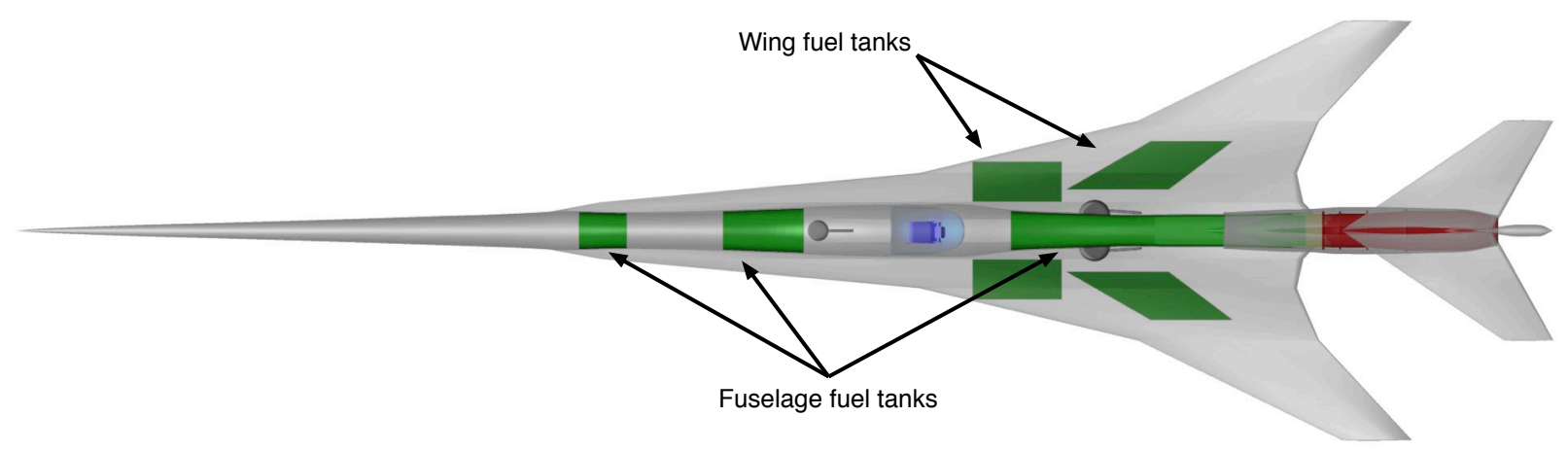

(b) Planform view showing fuselage and wing fuel tank locations

Figure 5. Internal placement of major components used to constrain the parametric model during shape optimization

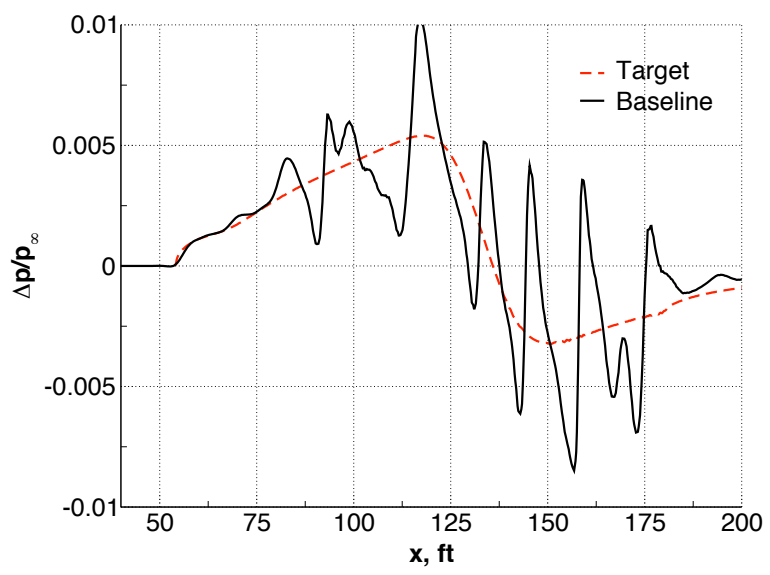

(a) Target and CFD-sampled baseline nearfield pressure signatures at $h / L=2$

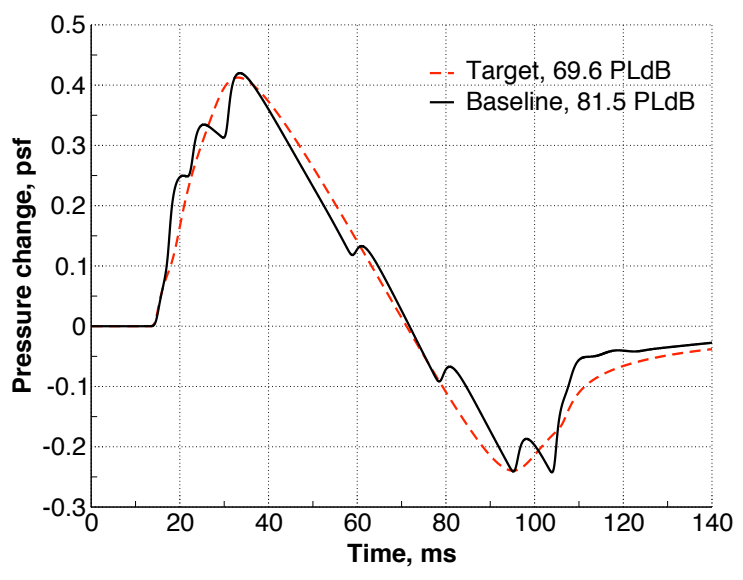

(b) Propagated ground signatures for target and baseline design

Figure 6. Nearfield pressure and ground signature propagated from $51,700 \mathrm{ft}$ for the optimization target and baseline design. $M_{\infty}=1.6$ 


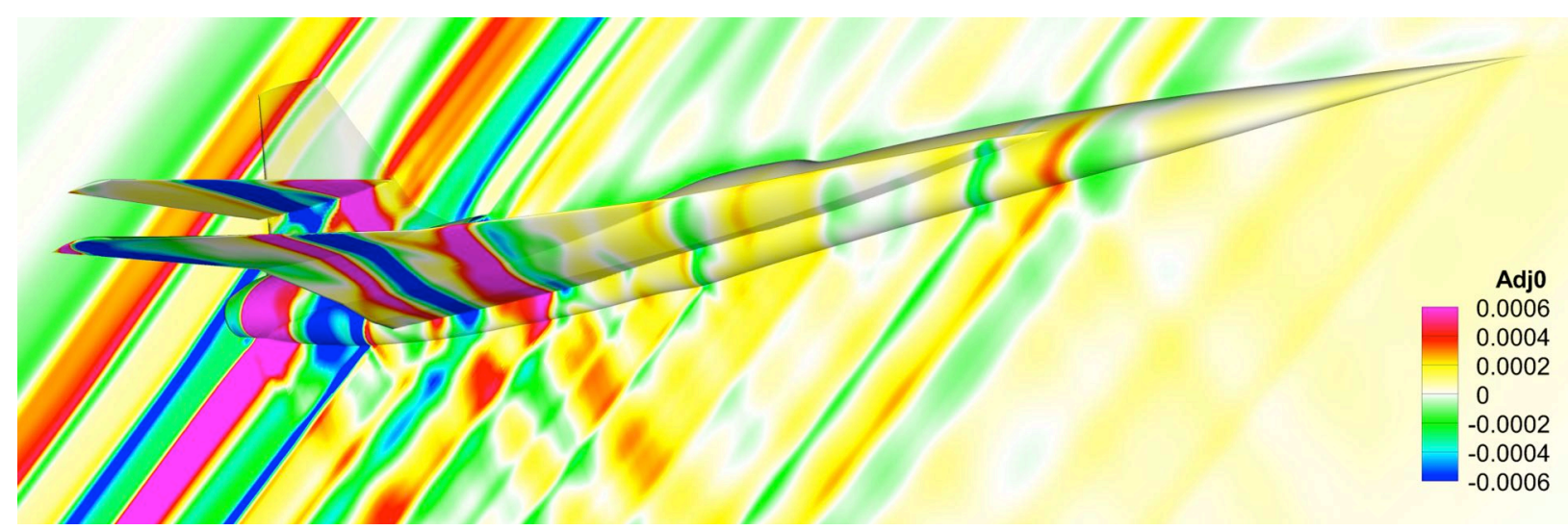

Figure 7. Contours of the density adjoint for the functional given in Eq. 1 plotted on the surface geometry and symmetry plane. $M_{\infty}=1.6, \alpha=3.375^{\circ}$

\section{III.A. Design Process Highlights}

In total, the shaping process consumed 46 design cycles. Several cycles, in particular those for which the parameterization was modified, are selected for further discussion. Along with plots of corresponding nearfield pressure and ground signature, the trim state, inviscid L/D, and predicted ground signature loudness are reported at each of these cycles.

\section{III.A.1. Initial Shaping}

To this point, the optimization was allowed to vary the forward fuselage, outer three wing sections and horizontal tail parameters. While such aft tailoring is affected by later modifications to upstream geometry, experience has shown that early mitigation of strong shocks using lifting surfaces leads to smoother surface features less likely to exploit shock cancellation.

Design cycle 6 represents the point at which the optimizer was no longer able to make further progress using the initial model parameterization. The nearfield pressure sampled at this cycle is shown in Fig. 8(a), and the propagated ground signature is shown in Fig. 8(b). Relative to the baseline, three of the four

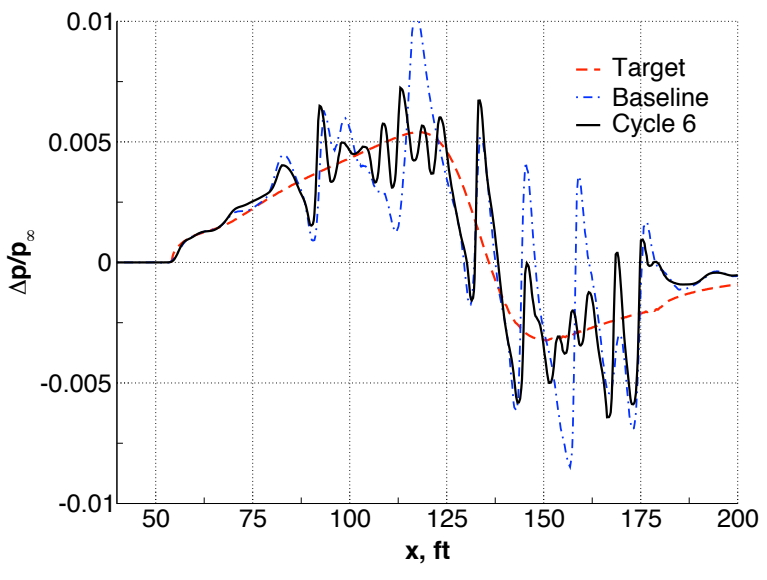

(a) Nearfield pressure signatures sampled at $h / L=2$

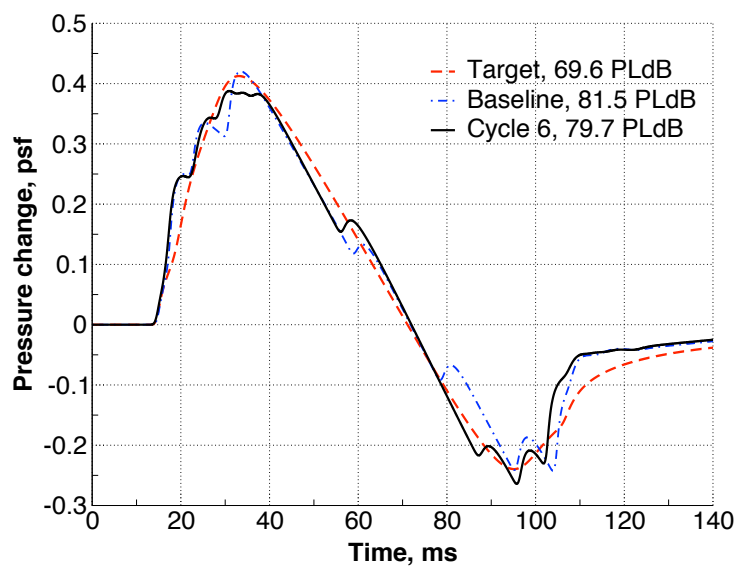

(b) Propagated ground signatures

Figure 8. Nearfield pressure and ground signature propagated from the $51,700 \mathrm{ft}$ cruise condition for the cycle 6 and baseline designs. $M_{\infty}=1.6$

strongest shocks have been appreciably diminished in strength. However, the current parameterization is unable to control the oscillation at $x \approx 85 \mathrm{ft}$, corresponding roughly with the fuselage and wing root leading edge intersection. At this cycle, $x_{\mathrm{cp}}=81.2 \mathrm{ft}$, inviscid L/D $=6.35$, and predicted loudness is 79.7 PLdB. 


\section{III.A.2. Fuselage Re-Parameterization}

Three additional fuselage sections were introduced, and existing sections re-distributed to coincide with regions of high sensitivity. Over the next several cycles, the fuselage and nacelle parameters were activated in groups, starting at the nose and sweeping aft. The nearfield pressure sampled at cycle 15, along with the propagated ground signature are shown in Fig. 9(a) and (b), respectively. The updated fuselage parame-

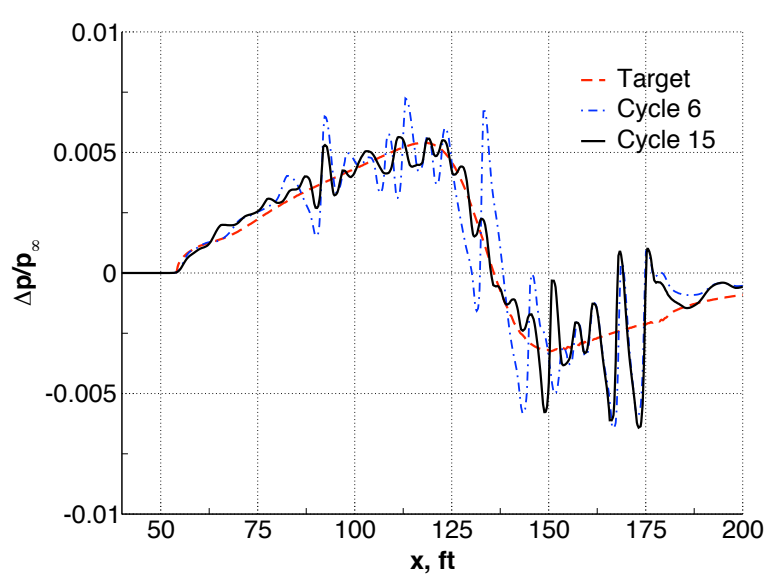

(a) Nearfield pressure signatures sampled at $h / L=2$

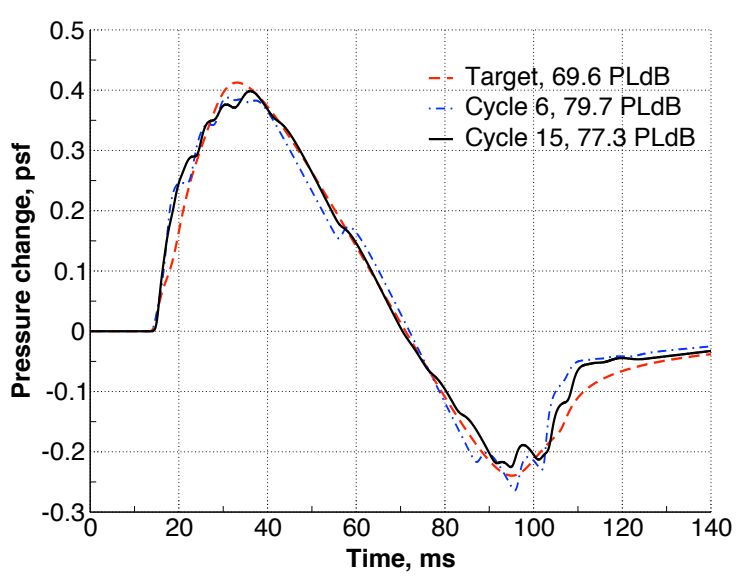

(b) Propagated ground signatures

Figure 9. Nearfield pressure and ground signature propagated from the $51,700 \mathrm{ft}$ cruise condition for the cycle 15 and cycle 6 designs. $M_{\infty}=1.6$

terization, coupled with the expanded design variable set, enables the optimizer to more effectively control deviations from the target signature. In particular, the oscillation at $x \approx 85 \mathrm{ft}$ is reduced in strength, and the remaining strong expansion shock at $x \approx 130 \mathrm{ft}$ is largely eliminated. At this cycle, $x_{\mathrm{cp}}=80.9 \mathrm{ft}$, inviscid $\mathrm{L} / \mathrm{D}=6.1$, and predicted loudness is $77.3 \mathrm{PLdB}$.

The remaining aft shock system starting at $x \approx 150 \mathrm{ft}$ could not be improved upon without further changes to the parameterization. Figure 10 shows the high adjoint sensitivities in this region associated with the horizontal tail and vertical tail tip.

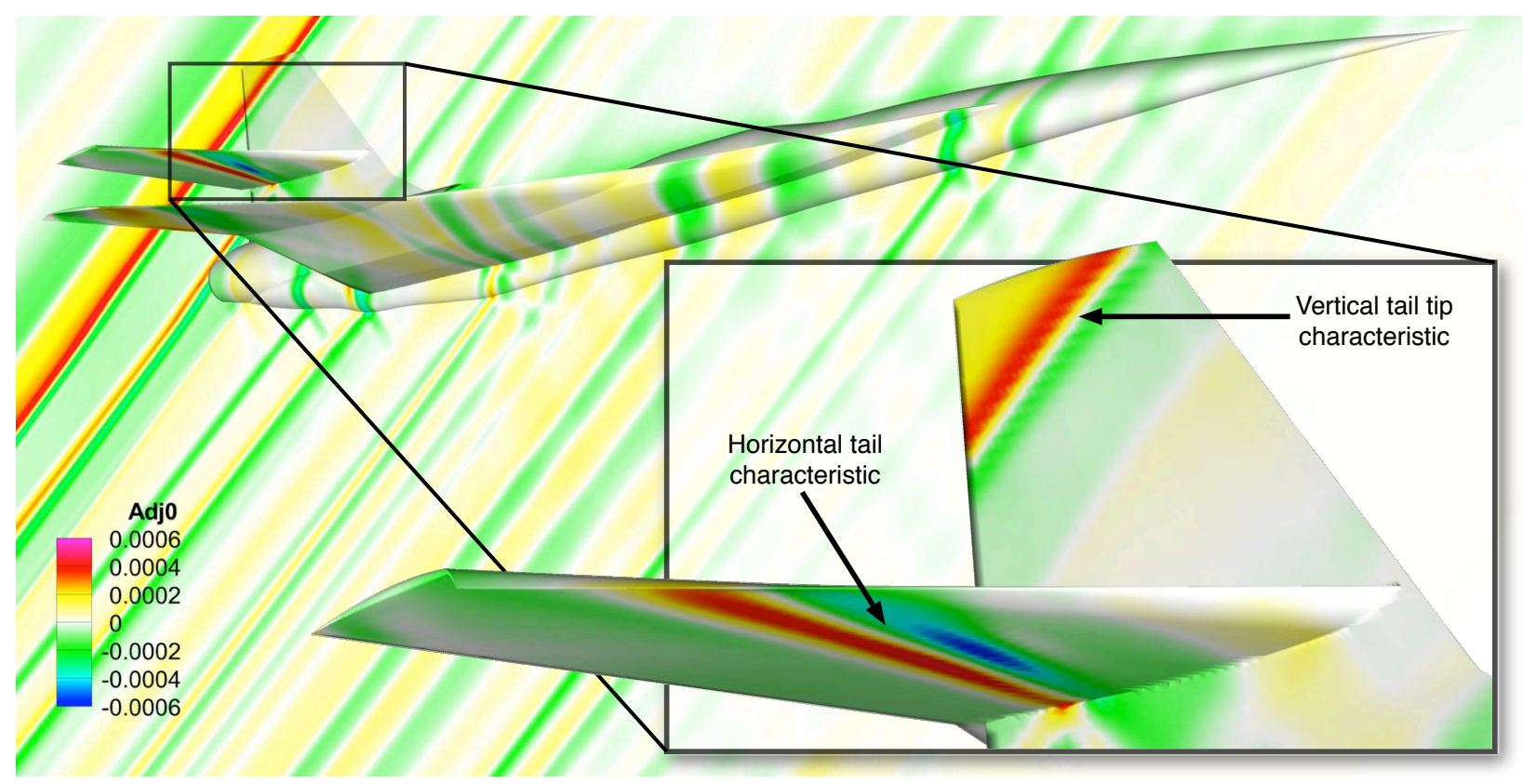

Figure 10. Contours of the density adjoint plotted on the geometry and symmetry plane at cycle 15; inset identifies the regions of high sensitivity on the horizontal and vertical tails. $M_{\infty}=1.6, \alpha=3.375^{\circ}$ 


\section{III.A.3. Tail Pod Addition}

To reduce sensitivities associated with the horizontal tail characteristic, an additional section was inserted at mid-span. At the tip of the vertical tail, an axisymmetric tail pod was introduced. The pod radius was allowed to vary at five stations, uniformly distributed about its axis, as depicted in Fig. 11. Pod axial position and length were also allowed to vary.

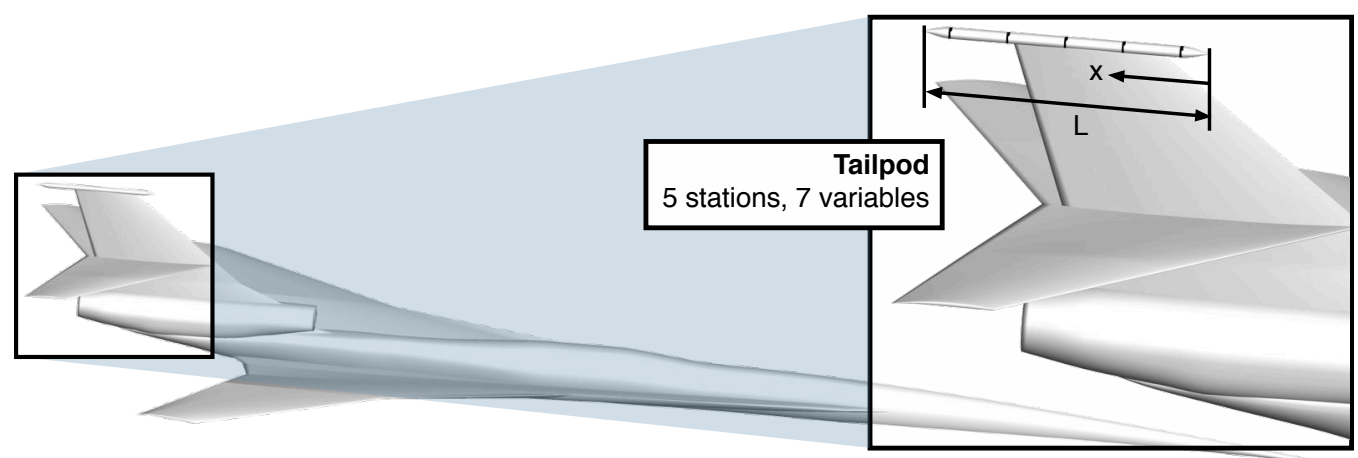

Figure 11. Tailpod component introduced to control shock associated with vertical tail

Optimization of the pod and horizontal tail geometry yielded the nearfield pressure sampled at cycle 19, shown in Fig. 12(a). The propagated ground signature is shown in Fig. 12(b). The amplitudes of the

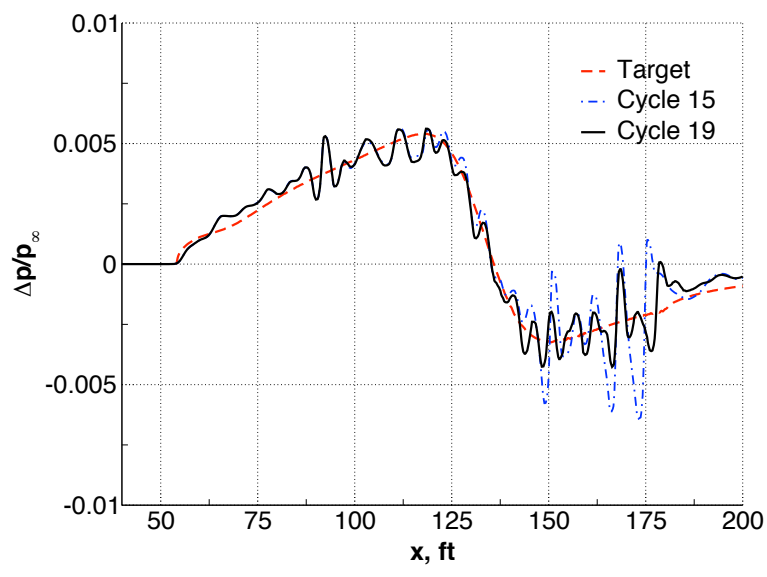

(a) Nearfield pressure signatures sampled at $h / L=2$

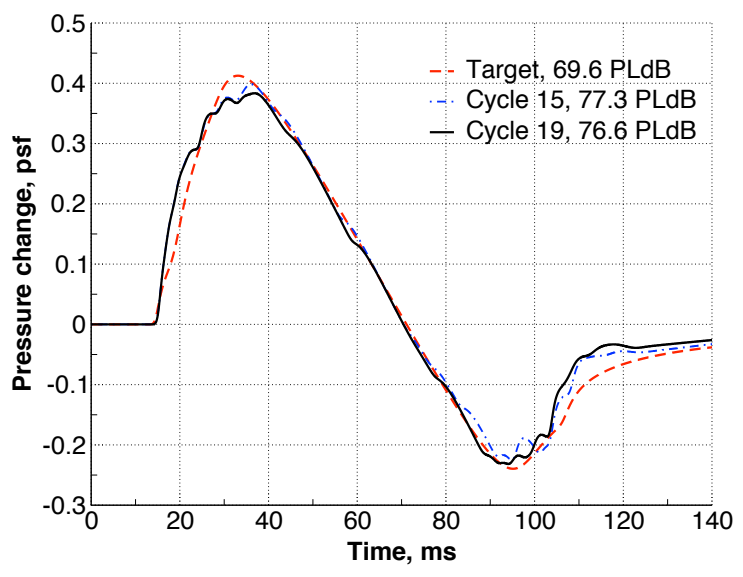

(b) Propagated ground signatures

Figure 12. Nearfield pressure and ground signature propagated from the 51,700 $\mathrm{ft}$ cruise condition for the cycle 19 (with tailpod) and cycle 15 (prior to tailpod addition) designs. $M_{\infty}=1.6$

system of aft shocks is reduced, and the adjoint field, shown in Fig. 13, has weakened globally. At this cycle, $x_{\mathrm{cp}}=81.2 \mathrm{ft}$, inviscid L/D $=6.7$, and predicted loudness is 76.6 PLdB.

At this point, a more refined main gear model - in particular one more carefully reproducing the articulation of the gear as it retracted into the body - was obtained. Fitment checks revealed that the as-designed OML would not fully encapsulate the retracted gear, requiring the introduction of a main gear fairing.

\section{III.A.4. Main Gear Integration}

The presence of the fairing, shown in Fig. 14(a), resulted in substantial degradation of the nearfield pressure signature, with predicted loudness of the propagated sonic boom rising to $79.2 \mathrm{PLdB}$. Local re-tailoring of the fairing and fuselage shapes, while successfully recovering favorable boom characteristics, resulted in unacceptably large oscillations of the OML about the fairing, as seen in Fig. 14(b).

To increase degrees of freedom available during shaping, the two Kulfan-parameterized inboard wing sections were re-activated. In addition, leveraging ongoing refinements to the target-generation capability, ${ }^{39}$ 


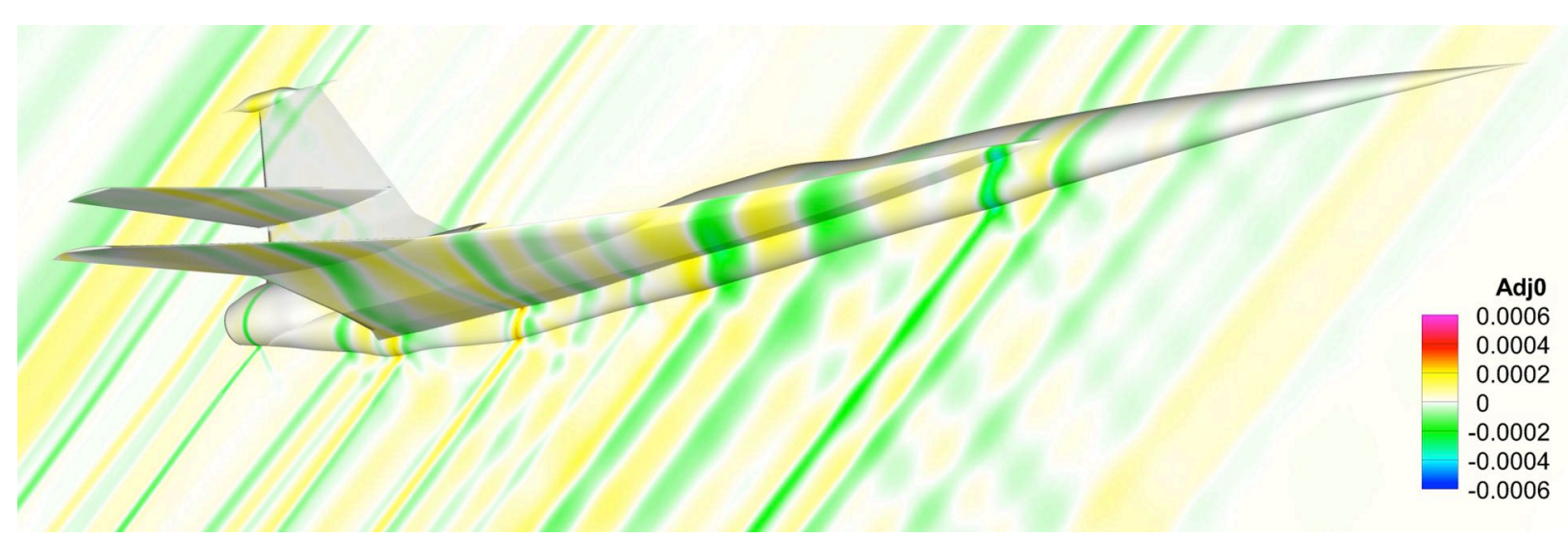

Figure 13. Contours of the density adjoint plotted on the cycle 19 surface geometry and symmetry plane. $M_{\infty}=1.6, \alpha=3.375^{\circ}$
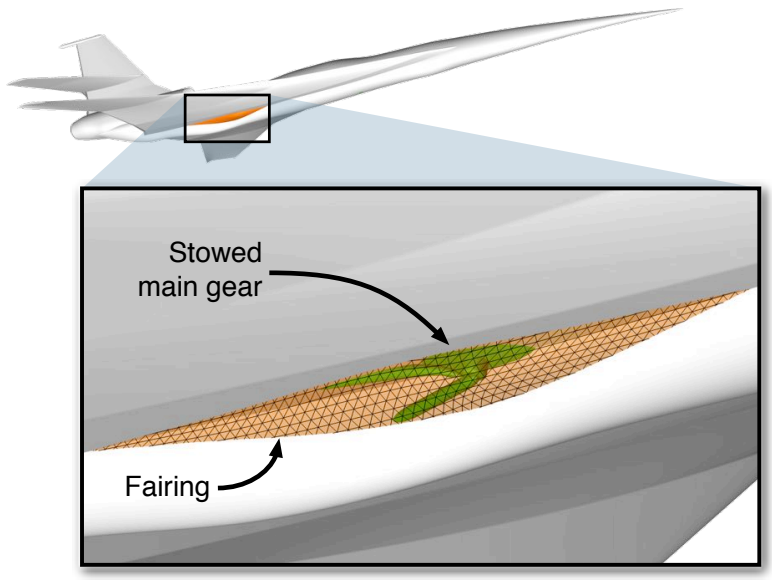

(a) Detail view of main gear OML protrusion in stowed state, with added fairing

Figure 14. Illustration of main landing gear fairing and resulting pathological OML geometry following fuselage shaping

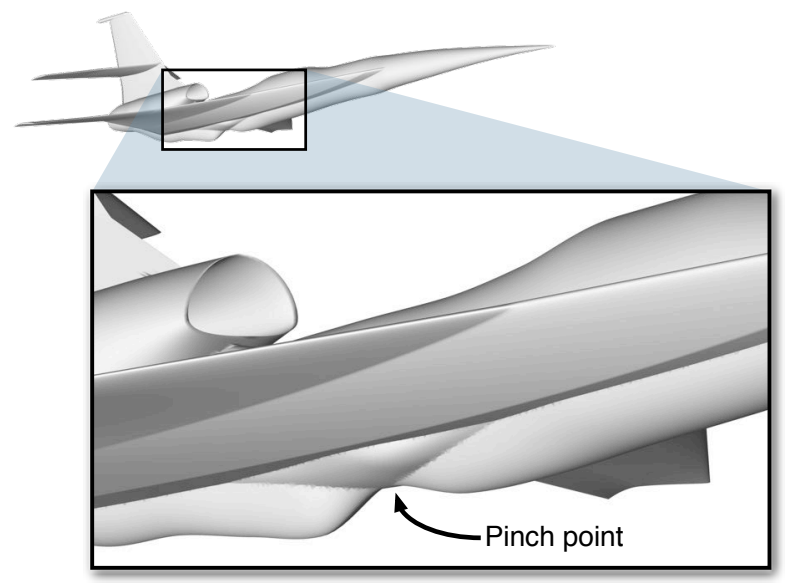

(b) Detail view of OML "pinch point" occurring when reshaping the fuselage in the presence of the fairing 
a new target pressure signature was produced. In addition to better accommodating the current under-track $A_{e}$, target loudness was reduced by $\sim 3$ PLdB to 66.7 PLdB.

The shaping strategy used by the optimizer was unexpected. Rather than applying a combination of fuselage, fairing, and wing shaping, the optimizer instead elected to focus on the wing root, thickening it until it swallowed the fairing entirely. This evolution happened within one design cycle (cycle 30), for which convergence history and geometry state at select iterations are shown in Fig. 15. While appearing dramatic,

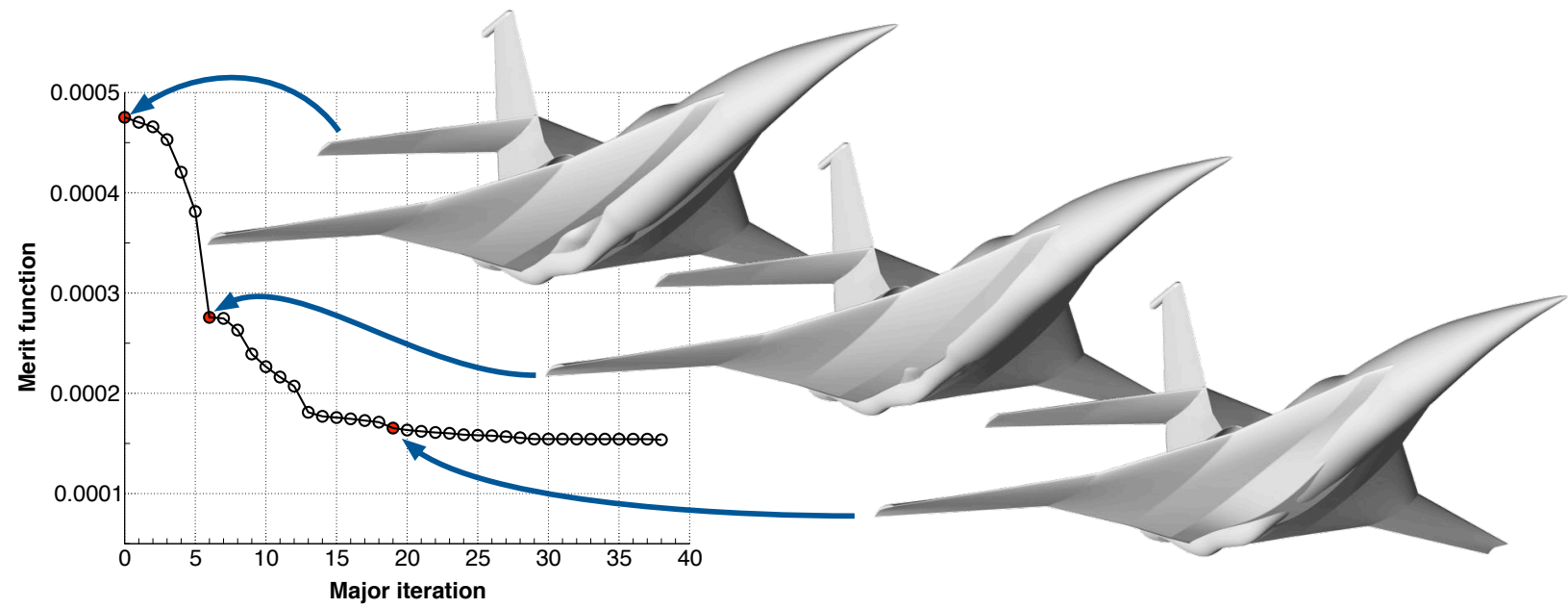

Figure 15. Convergence history for design cycle 30, with OML evolutions illustrated for the initial state, after 6 iterations, and after 19 iterations. Subsumption of the fairing by the wing root is essentially complete by the 15th iteration

a plot of the component cross-sections for the cycle 30 optimized geometry in Fig. 16 shows that actual volume addition at any given location is in fact minimal, with the wing root closely following the fuselage contours.

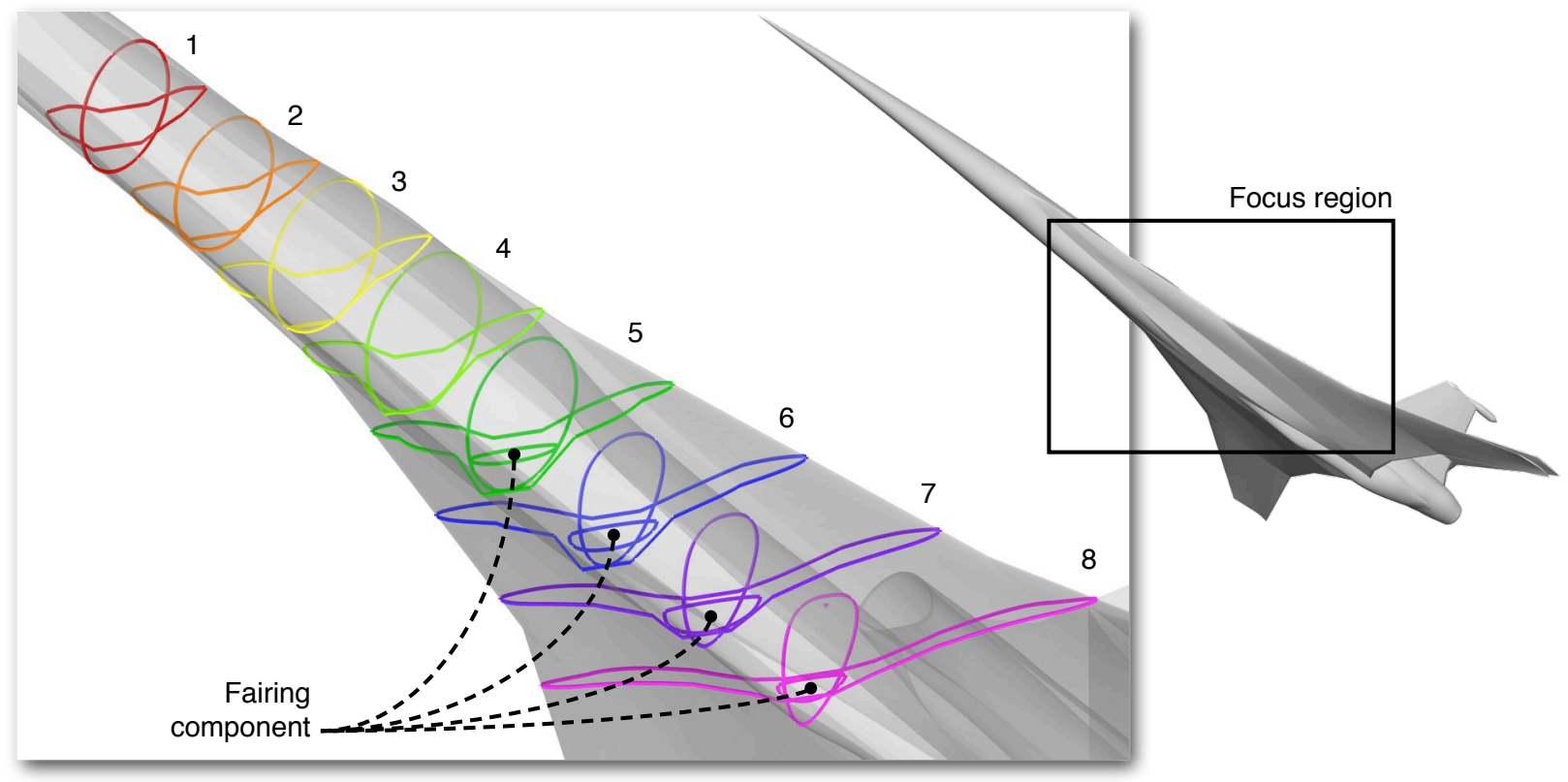

Figure 16. Component cross-sections sampled at $5 \mathrm{ft}$ intervals within the indicated region. Sections 3-5 illustrate how closely the wing surface follows that of the fuselage, while section 7 shows the wing surface just outside of the fairing component

Coupled with the updated target, and following careful re-application of design lessons learned to this point, the cycle $37 \mathrm{OML}$ was felt to be a reasonable point at which to terminate shaping. The nearfield pressure and propagated ground signatures for this design are shown in Fig. 17. As a point of comparison, 
the cycle 29 signal is included to illustrate the signal degradation that resulted from addition of the fairing. At this cycle, $x_{\mathrm{cp}}=81.3 \mathrm{ft}$, inviscid L/D $=6.6$, and predicted loudness is $71.8 \mathrm{PLdB}$. Note that the key

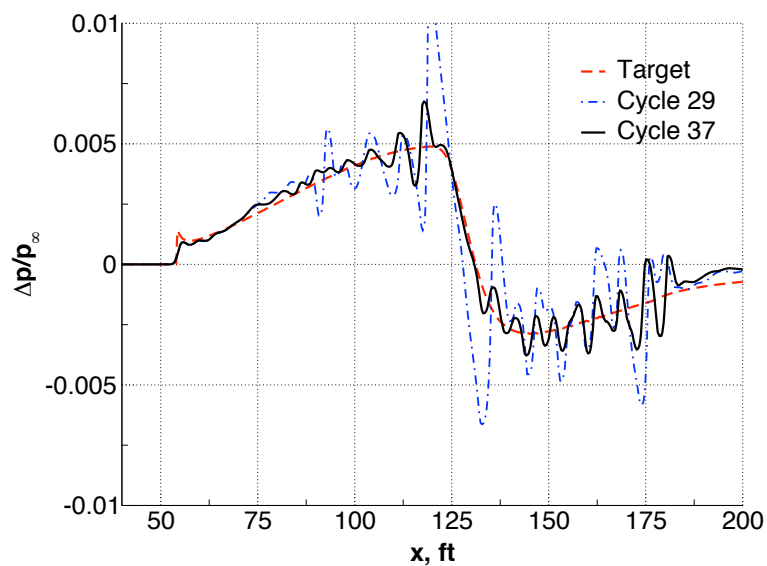

(a) Nearfield pressure signatures sampled at $h / L=2$

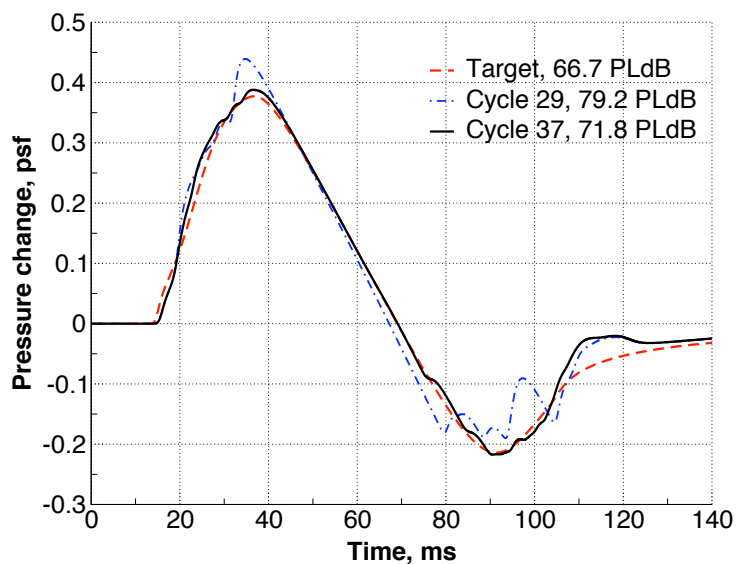

(b) Propagated ground signatures

Figure 17. Nearfield pressure and ground signature propagated from the 51,700 $\mathrm{ft}$ cruise condition for the cycle 37 and cycle 29 designs. $M_{\infty}=1.6$

result is not the loudness level achieved, but rather the almost 10 PLdB reduction relative to the starting point.

\section{III.A.5. Propagation from Increased Offset Distances}

To determine a more appropriate standoff distance for propagation, an adaptive refinement study using the cycle 37 OML was performed. Targets were specified at $h / L=\{1,2, \ldots, 5\}$, and the mesh was allowed to grow to 380 million cells. Figure 18(a) shows plots of stacked nearfield pressure signatures sampled from the terminal mesh, with corresponding propagated ground signals plotted in Fig. 18(b). In the nearfield,

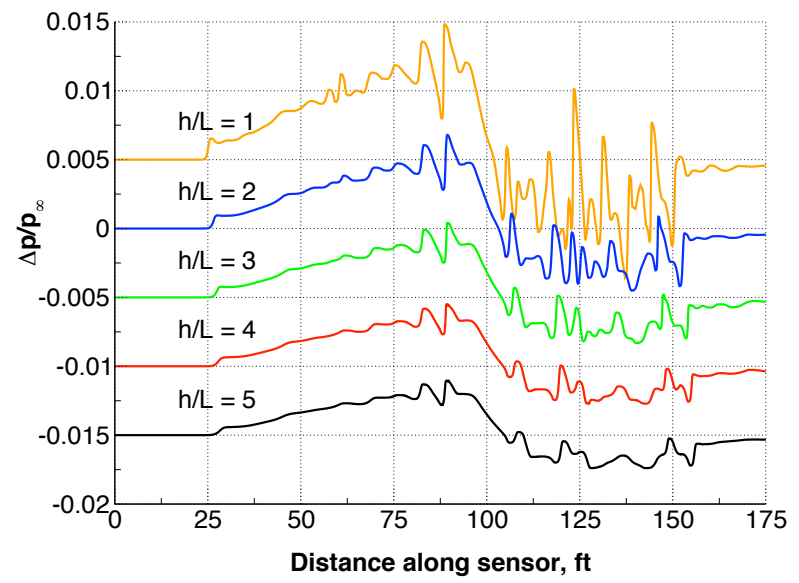

(a) Nearfield pressure signatures sampled at $h / L=$ $\{1,2, \ldots, 5\}$

Figure 18. Nearfield pressures and ground signatures propagated from the 51,700 $\mathrm{ft}$ cruise condition for the cycle 38 design. $M_{\infty}=1.6$

changes in the aft signal structure suggest that, from $h / L=1$ to $h / L=2$, shock interactions are continuing to occur that would invalidate the axisymmetric assumption for propagation. The $h / L=3$ to $h / L=5$ curves are notable for their similarity, with attenuation being the dominant effect. The ground signatures change qualitatively from $h / L=1$ through $h / L=3$, with visible differences in the aft signal shape. Specifically, 
the shock at $\sim 90 \mathrm{~ms}$ is missed at $h / L=1$, and the shock just after the 100 ms mark continues to grow in amplitude until $h / L=3$. Predicted loudness increases from $75.1 \mathrm{PLdB}$ at $h / L=1$ to $76.1 \mathrm{PLdB}$ at $h / L=3$. It is essentially unchanged at $h / L=4$, then decreases slightly to $75.7 \mathrm{PLdB}$ at $h / L=5$, with the falloff felt to be an artifact of increasing nearfield signature dissipation with standoff distance. Taken as a whole, these results indicate that for this configuration, an offset distance of $h / L=3$ is consistent with propagation theory.

\section{III.A.6. Refine at $h / L=3$}

The design mesh was revised to accommodate a target at $h / L=3$. Regions of high refinement were expanded to encapsulate the zone of influence between vehicle and sensor, resulting in a mesh containing 60 million cells. Function evaluation times - that is, time for a flow and adjoint solve - increased by a factor of $7^{\mathrm{a}}$.

Shape changes made by the optimizer during this refinement cycle were small, with design sensitivity limited primarily to the nacelle and tail regions. The nearfield pressure and propagated ground signatures for the cycle 38 design are shown in Fig. 19. The cycle 37 pressures sampled at and propagated from $h / L=3$ are included for comparison. Consistent with the subtle OML changes, nearfield pressure changes were small

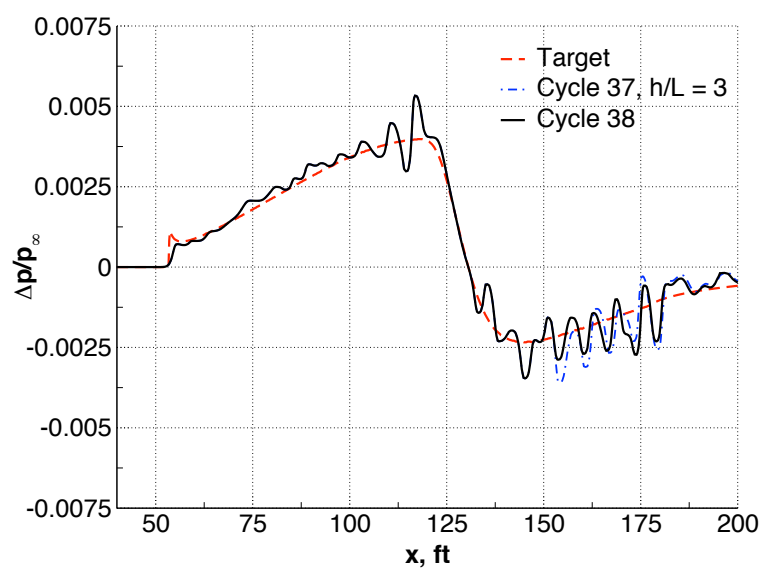

(a) Nearfield pressure signatures sampled at $h / L=3$

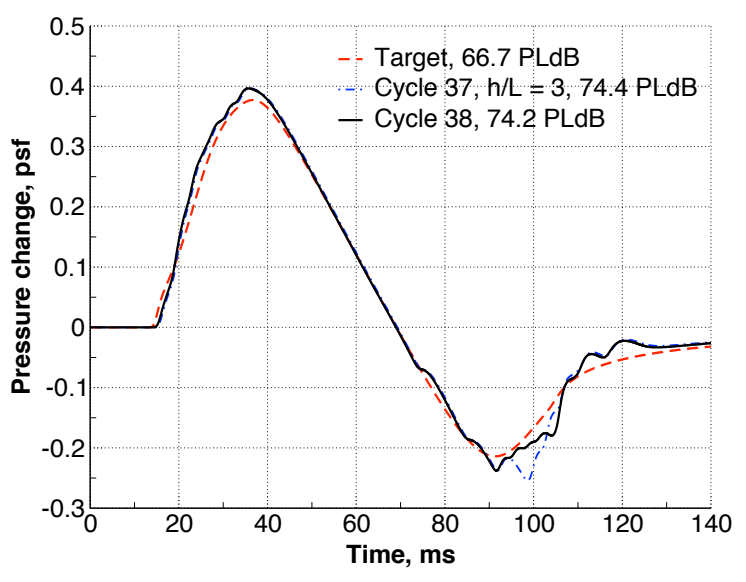

(b) Propagated ground signatures

Figure 19. Nearfield pressure and ground signature propagated from the 51,700 $\mathrm{ft}$ cruise condition for the cycle 37 and 38 designs. $M_{\infty}=1.6$

and confined to the aft region of the signal. Similarly, predicted loudness is essentially unchanged, with only a $0.2 \mathrm{PLdB}$ drop to $74.2 \mathrm{PLdB}$ for the cycle 38 design. The increase in loudness when the cycle 37 OML is propagated from $h / L=3$ is larger than expected, and is an artifact of the aggressive $h / L=2$ mesh construction. However, similarity between the cycle 37 and 38 OMLs indicate that it is effective for initial shaping, with only subtle re-tailoring of the OML required at $h / L=3$.

The adjoint field for the cycle 38 geometry is shown in Fig. 20. Relative to the cycle 19 field shown in Fig. 13, the general diminishing of field strength is primarily due to the increased offset distance of the target. The most notable difference is the elimination of the regions of finite sensitivity at the leading edge of the wing-fuselage intersection. At this cycle, $x_{\mathrm{cp}}=81.6 \mathrm{ft}$, inviscid $\mathrm{L} / \mathrm{D}=6.7$, and predicted loudness is 74.2 PLdB.

\section{III.B. Activation of Power Boundary Conditions}

Required cruise thrust was determined based on the CFD-predicted inviscid drag for the final flow-through OML, and a fully turbulent viscous drag estimate using the Sommer and Short $T^{\prime}$ method. ${ }^{40}$ Boundary condition (BC) parameters were produced using a NASA-developed NPSS ${ }^{11}$ (Numerical Propulsion System Simulation) engine deck for the F404-GE-402, with plenum and nozzle values selected to satisfy perfect plume expansion. Inflow BCs were specified at the fan face, with $p / p_{\infty}=2.32897, \rho / \rho_{\infty}=2.26651$, and

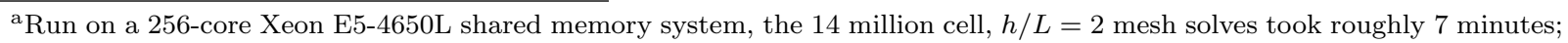
the $h / L=3,60$ million cell mesh solves took roughly 50 minutes. 


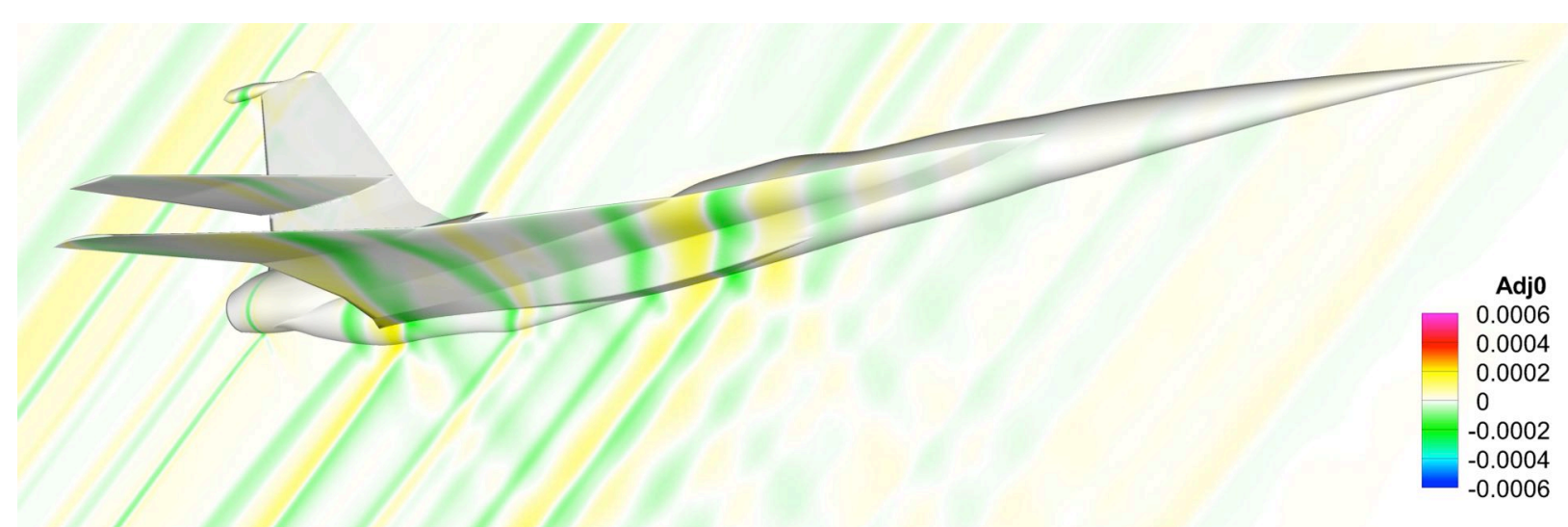

Figure 20. Contours of the density adjoint plotted on the cycle 38 surface geometry and symmetry plane. $M_{\infty}=1.6, \alpha=3.375^{\circ}$

$M=0.60929$ normal to the inflow face. The inflow area is $3.549 \mathrm{ft}^{2}$. Outflow BCs were specified at the plenum, with $p / p_{\infty}=10.0994, \rho / \rho_{\infty}=1.81048$, and $M=0.66196$ normal to the outflow face. The plenum, throat, and exit areas are $4.203 \mathrm{ft}^{2}, 1.725 \mathrm{ft}^{2}$, and $4.459 \mathrm{ft}^{2}$, respectively.

Given the need to control dissipation between vehicle and offbody sensor, the 60 million cell flow-through design mesh already included regions of high refinement at the inlet and nozzle; this mesh construction was hence retained for design with power. Evaluating the as-designed OML with active power BCs revealed the introduction of a strong shock in the aft part of the nearfield signal, as shown in Fig. 21(a). The propagated ground signature is shown in Fig. 21(b), with predicted loudness of 80.7 PLdB, a more than 6 PLdB increase relative to the flow-through case.

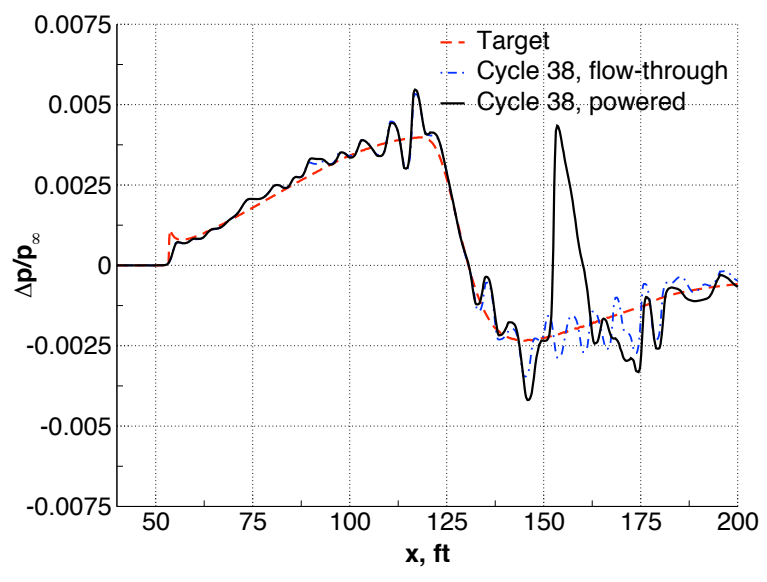

(a) Nearfield pressure signatures sampled at $h / L=3$

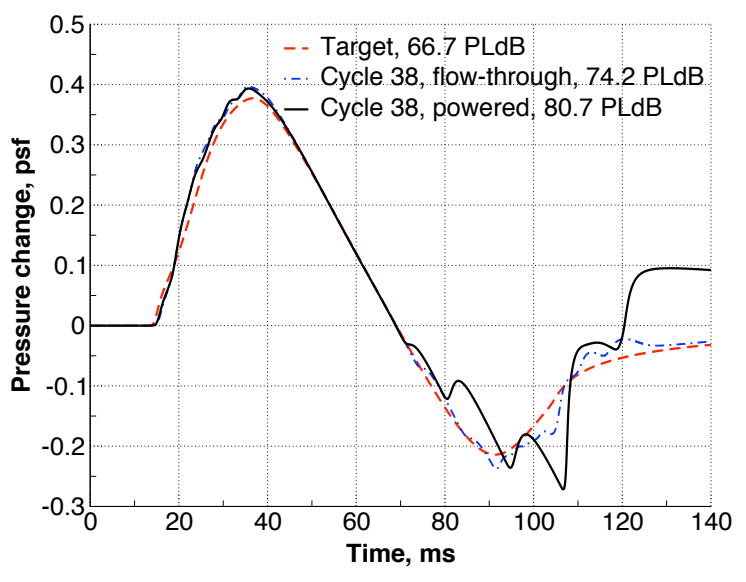

(b) Propagated ground signatures

Figure 21. Nearfield pressure and ground signature propagated from the 51,700 ft cruise condition for the cycle $38 \mathrm{OML}$ with and without active power BCs. $M_{\infty}=1.6$

The flow field effect of the active inflow and outflow faces is illustrated in Fig. 22, with contours of Mach number on the symmetry plane and coefficient of pressure $\left(C_{p}\right)$ on the body. Based on the Mach contours, the most notable changes are the spillage shock at the inlet, and the stronger oblique shock at the nozzle lip. In addition, an increase in the wing area washed over by the inlet shock is evident in the $C_{p}$ contours, with a corresponding $9 \%$ reduction in $C_{L}$ observed. Inlet spillage for the flow-through-designed configuration was determined to be in excess of $14 \%$. A parametric sweep study was used to relate inlet capture area to spillage for this geometry, enabling spillage to be reduced to $4 \%$. Inviscid L/D increased to 7.2 , and reported lift recovered to within $5 \%$ of the design value, with $C_{L}=0.062$. However, the effect on the nearfield pressure signal was negligible, and vehicle trim was compromised, with $x_{\mathrm{cp}}=82.7 \mathrm{ft}$. 


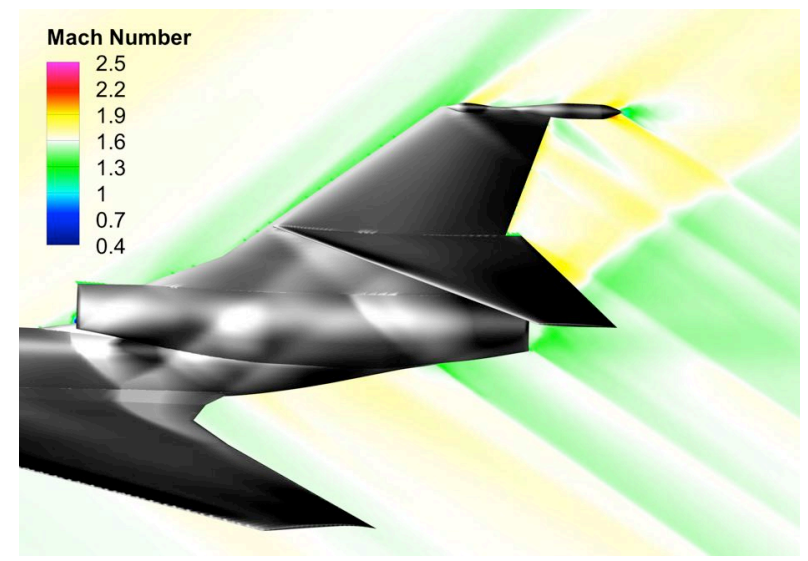

(a) Flow-through case

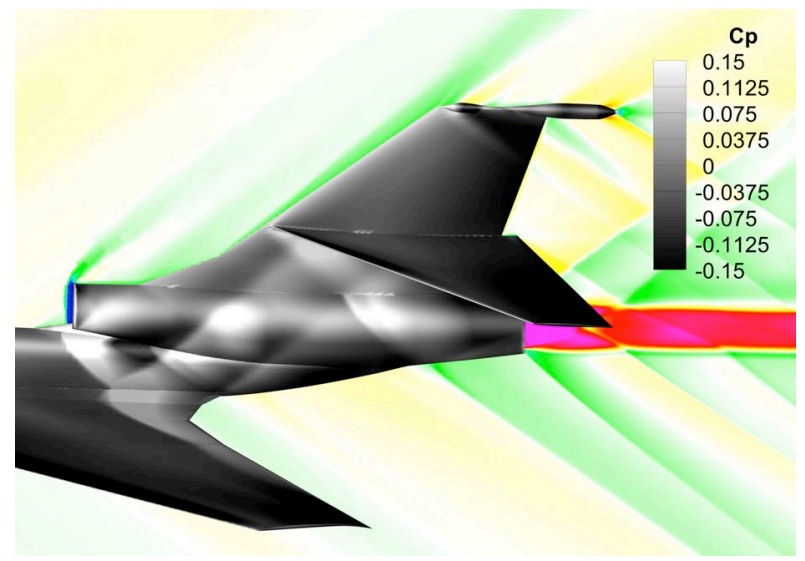

(b) Powered case

Figure 22. Comparison of flow-through and powered cases, with Mach contours plotted on the symmetry plane, and $C_{p}$ contours plotted on the body. $M_{\infty}=1.6, \alpha=3.375^{\circ}$

\section{III.B.1. Re-Shaping with Active Power BCs}

An adjoint sensitivity evaluation, focused about the strong aft shock, was used to guide selection of design parameters to activate during re-tailoring of the powered configuration. Figure 23(a) shows contours of density adjoint on the body and symmetry plane for the powered cycle 38 design, and the re-shaped cycle 41 design. The active target region is limited to $140<x<165 \mathrm{ft}$, and identified in the corresponding nearfield pressures, plotted in Fig. 23(b). This re-shaping was performed with the target at $h / L=2$, using a 14

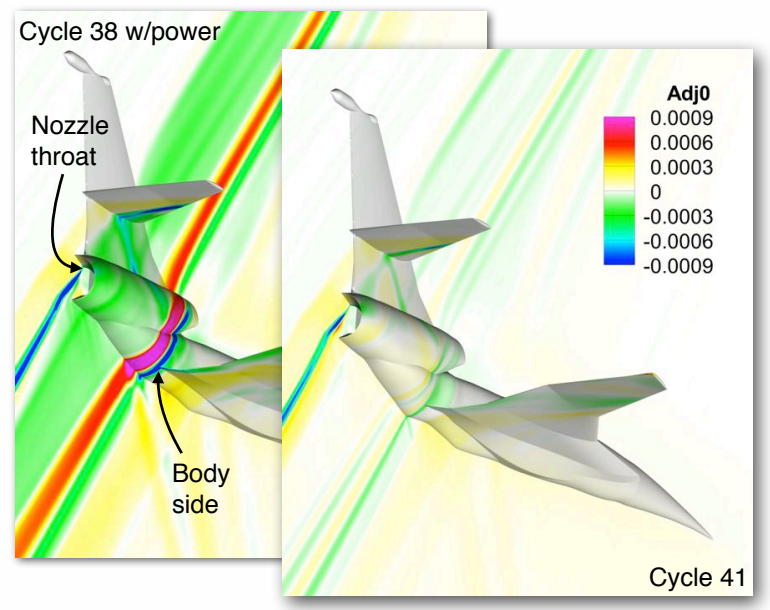

(a) Density adjoint contours with target active over interval $140<x<165 \mathrm{ft}$

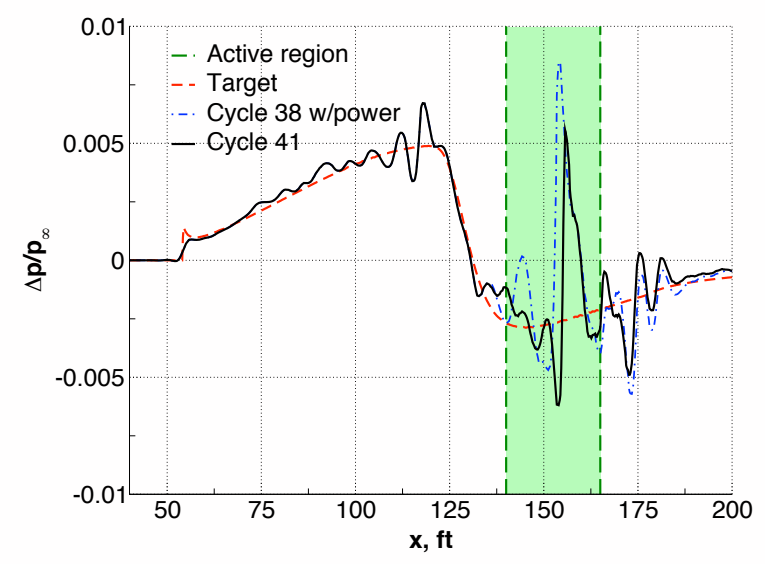

(b) Nearfield pressures sampled at $h / L=2$; shading indicates active target region

Figure 23. Comparison of initial powered results obtained on flow-through-optimized cycle 38 design, and at cycle 41 , following re-tailoring under power. $M_{\infty}=1.6, \alpha=3.375^{\circ}$

million cell mesh structure similar to that used during shaping of the flow-through design.

The region of high sensitivity at the body side identified in Fig. 23(a) is largely eliminated by cycle 41, along with the corresponding shock at $x \approx 140 \mathrm{ft}$. While the strong shock at $x \approx 150 \mathrm{ft}$ is weakened, its amplitude remains significant, and the propagated ground signature loudness is reduced by only 1 PLdB relative to the powered cycle 38 design. It is particularly interesting to note that, coinciding with this shock, the remaining region of high sensitivity can be traced almost entirely to the nozzle throat, where there is no design authority. 


\section{III.B.2. Transition to Plug Nozzle}

In Ref. 26, plug nozzles were shown to effectively mitigate the oblique shock occurring at the nozzle lip, with a longer, $10^{\circ}$ plug producing a noticeably weaker pressure disturbance than a shorter, $15^{\circ}$ plug. In this context, the plug itself also offers a mechanical means by which to shield the nozzle interior from the under-track observer. The existing converging-diverging (C-D) nozzle was replaced with a $12^{\circ}$ plug nozzle to explore whether it could be used to control the aft shock present with the current design. The C-D and plug nozzles are compared in Fig. 24. Relative to the C-D nozzle, the exit plane is moved aft by $1 \mathrm{ft}$, and the nozzle boat-tail angle is reduced from $7.7^{\circ}$ to $3.3^{\circ}$. Along with the plenum outflow face BCs, the plenum, throat and exit plane annuli are sized to match the areas of the C-D nozzle.

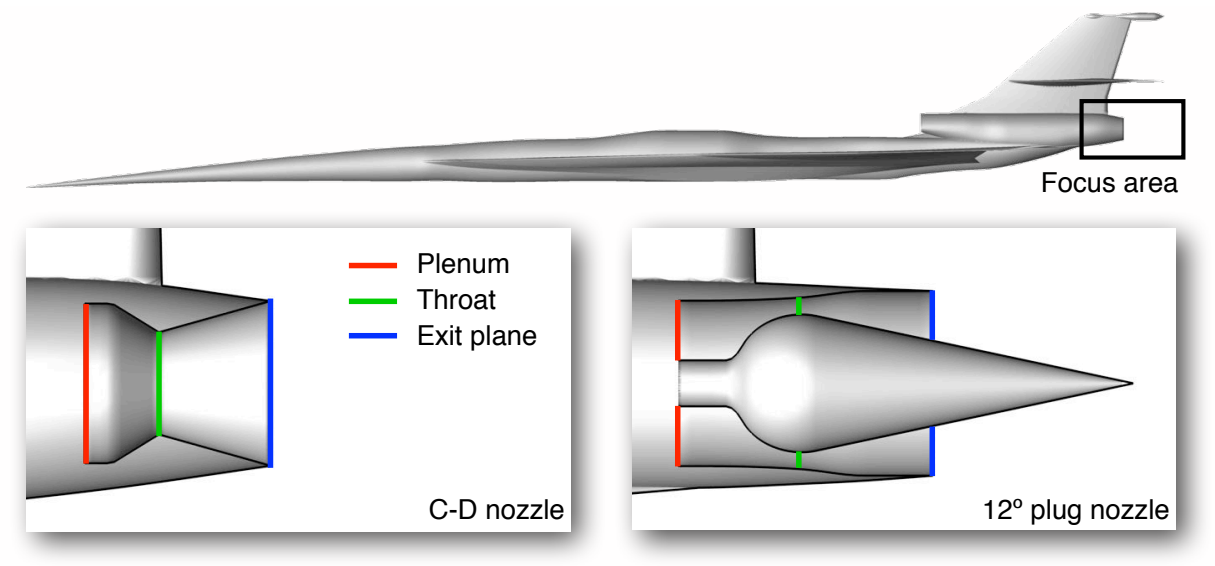

Figure 24. Comparison of conventional C-D and $12^{\circ}$ plug nozzles, with plenum, throat and exit plane equivalence as indicated

Contours of density adjoint for the cycle 41 OML with the plug nozzle are plotted in Fig. 25(a). The result is promising: unlike the C-D nozzle case, the region of high sensitivity has migrated from the nozzle interior to the lower cowl surface. A comparison of the nearfield pressures sampled for the cycle $41 \mathrm{OML}$ with C-D and plug nozzles is given in Fig. 25(b). Prior to any re-shaping, the strength of the shock at $x \approx 150 \mathrm{ft}$ has been reduced; however an additional shock of similar strength appears at $x \approx 165 \mathrm{ft}$.

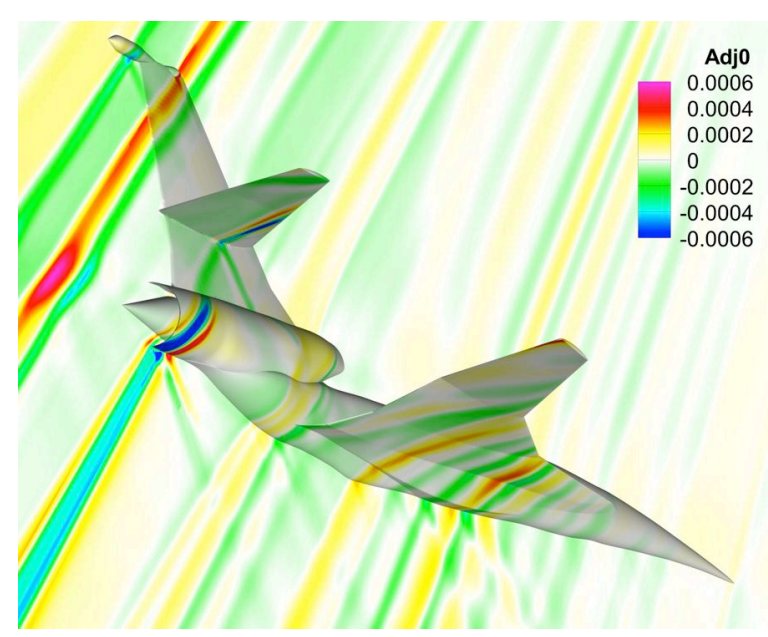

(a) Density adjoint contours for cycle 41 OML with $12^{\circ}$ plug nozzle

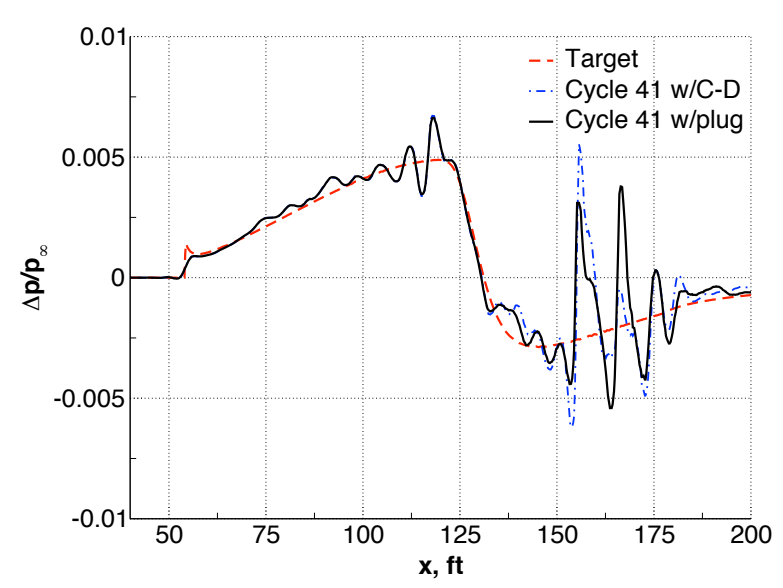

(b) Nearfield pressures sampled at $h / L=2$

Figure 25. Comparison of cycle $41 \mathrm{OML}$ with $\mathrm{C}-\mathrm{D}$ and $12^{\circ}$ plug nozzles. $M_{\infty}=1.6, \alpha=3.375^{\circ}$

Over the next three design cycles, shape parameters affecting the aft fuselage, nacelle, nozzle cowl, and tailpod were activated. Consistent with the flow-through shaping methodology, initial shaping was performed with the smaller, $h / L=2$ mesh, then final refinement was performed on the larger, $h / L=3$ mesh. 
The nearfield pressure for the re-shaped, cycle 44 design is plotted in Fig. 26(a), while the corresponding propagated ground signature is shown in Fig. 26(b).

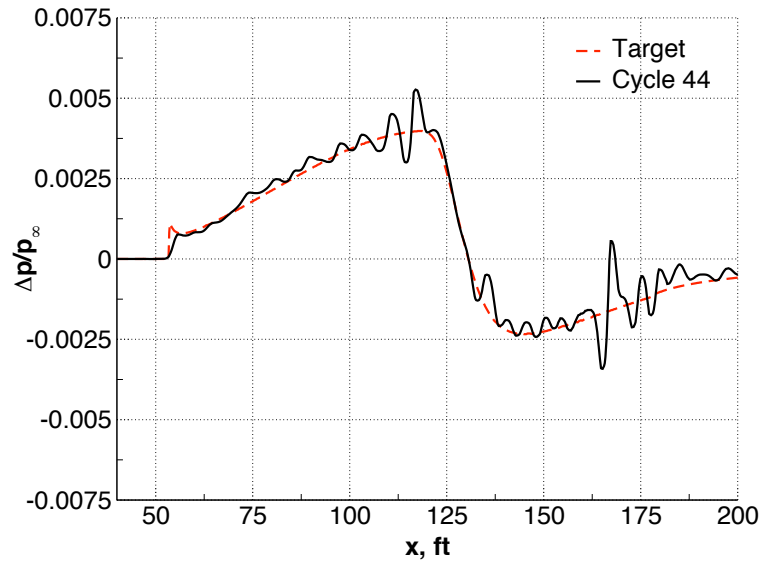

(a) Nearfield pressure signature sampled at $h / L=3$

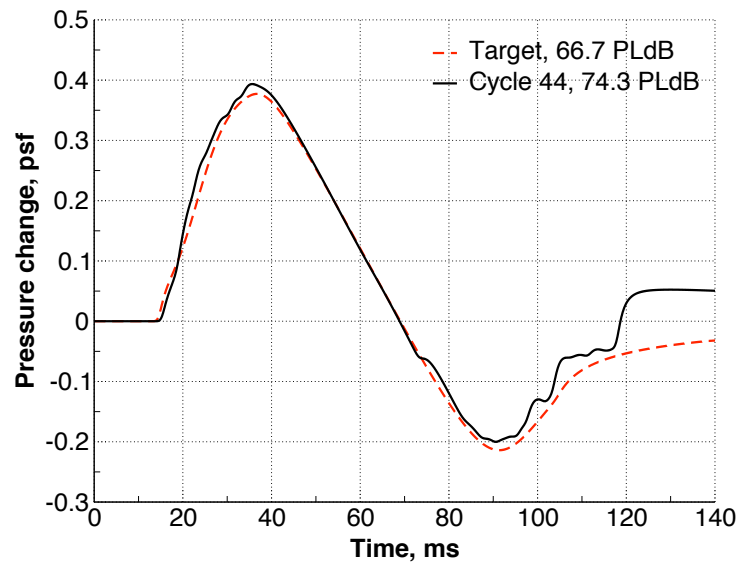

(b) Propagated ground signature

Figure 26. Nearfield pressure and ground signature propagated from the 51,700 ft cruise condition for the cycle 44 OML. $M_{\infty}=1.6$

Density adjoint contours for the cycle 44 geometry are shown in Fig. 27. In addition to general weakening of the adjoint field, the regions of high sensitivity about the plug nozzle and tailpod evident on the cycle 41 geometry have been largely eliminated. At this cycle, $x_{\mathrm{cp}}=81.1 \mathrm{ft}$, inviscid L/D $=6.0$, and predicted

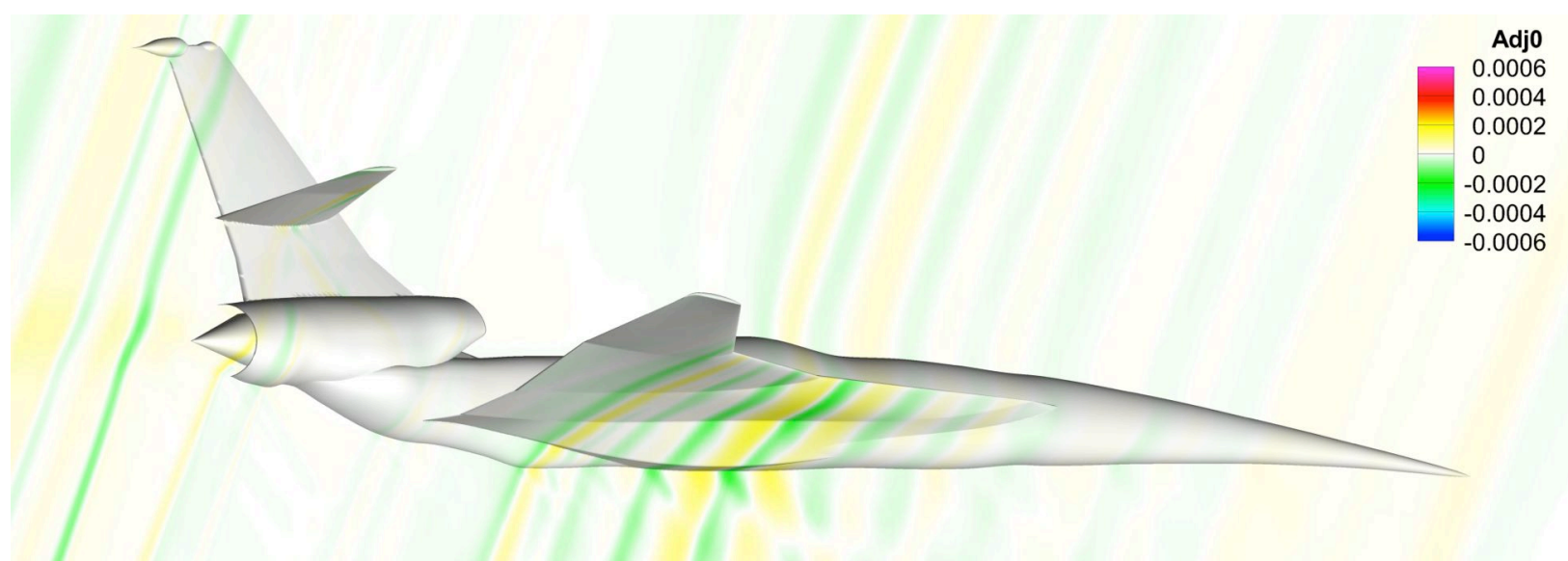

Figure 27. Contours of the density adjoint plotted on the cycle 44 surface geometry and symmetry plane. $M_{\infty}=1.6, \alpha=3.375^{\circ}$

loudness is $74.3 \mathrm{PLdB}$. While trim has recovered, the marked reduction in inviscid L/D is related to a $17 \%$ lift deficit, with reported $C_{L}$ falling to 0.055 .

\section{III.B.3. Applying Mesh Refinement During Design}

The cycle 44 design was evaluated using an adaptively refined mesh to determine if boom performance persisted under high resolution analysis. As shown in Fig. 28(a), the design mesh was unable to capture a significant aft shock. Loudness predicted from the propagated ground signature, shown in Fig. 28(b), increased by $9 \mathrm{PLdB}$, to $83.3 \mathrm{PLdB}$. Figure 29 compares mesh structure and pressure contours in the near-body and nearfield regions of the pre-specified and adapted meshes. While superficially similar, pressure contours on the adaptively refined mesh are both more sharply resolved and differ in character, particularly near the symmetry plane, relative to the pre-specified mesh result. The $z=70 \mathrm{ft}$ plane on which pressure contours are plotted coincides with the nozzle exit; mesh adaptation patterns suggest this region as being a prime contributor to the shock. 


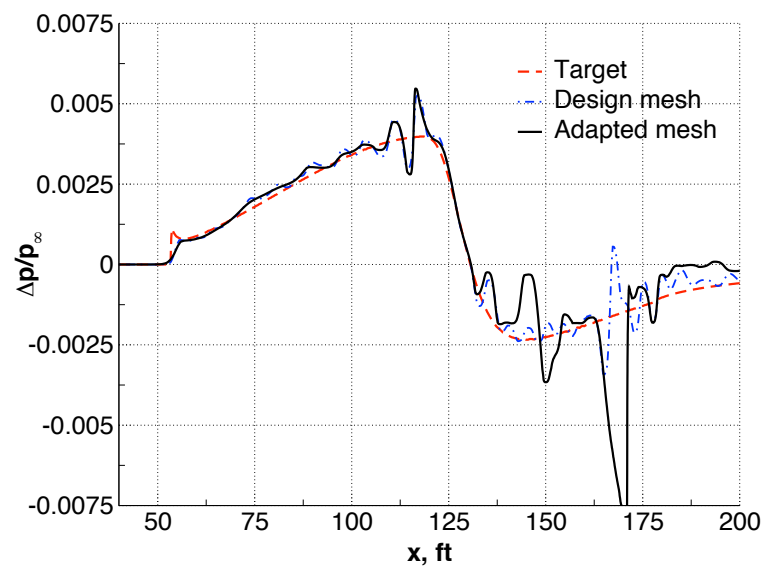

(a) Nearfield pressure signatures sampled at $h / L=3$

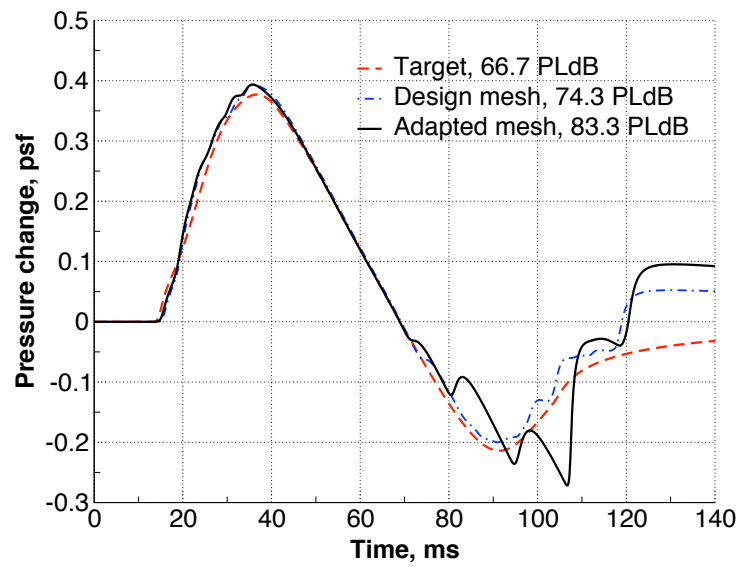

(b) Propagated ground signatures

Figure 28. Nearfield pressure and ground signature propagated from the 51,700 $\mathrm{ft}$ cruise condition for the cycle $44 \mathrm{OML}$. The adaptively refined mesh contained 92 million cells; the 56 million cell design mesh solution is shown for reference. $M_{\infty}=1.6$
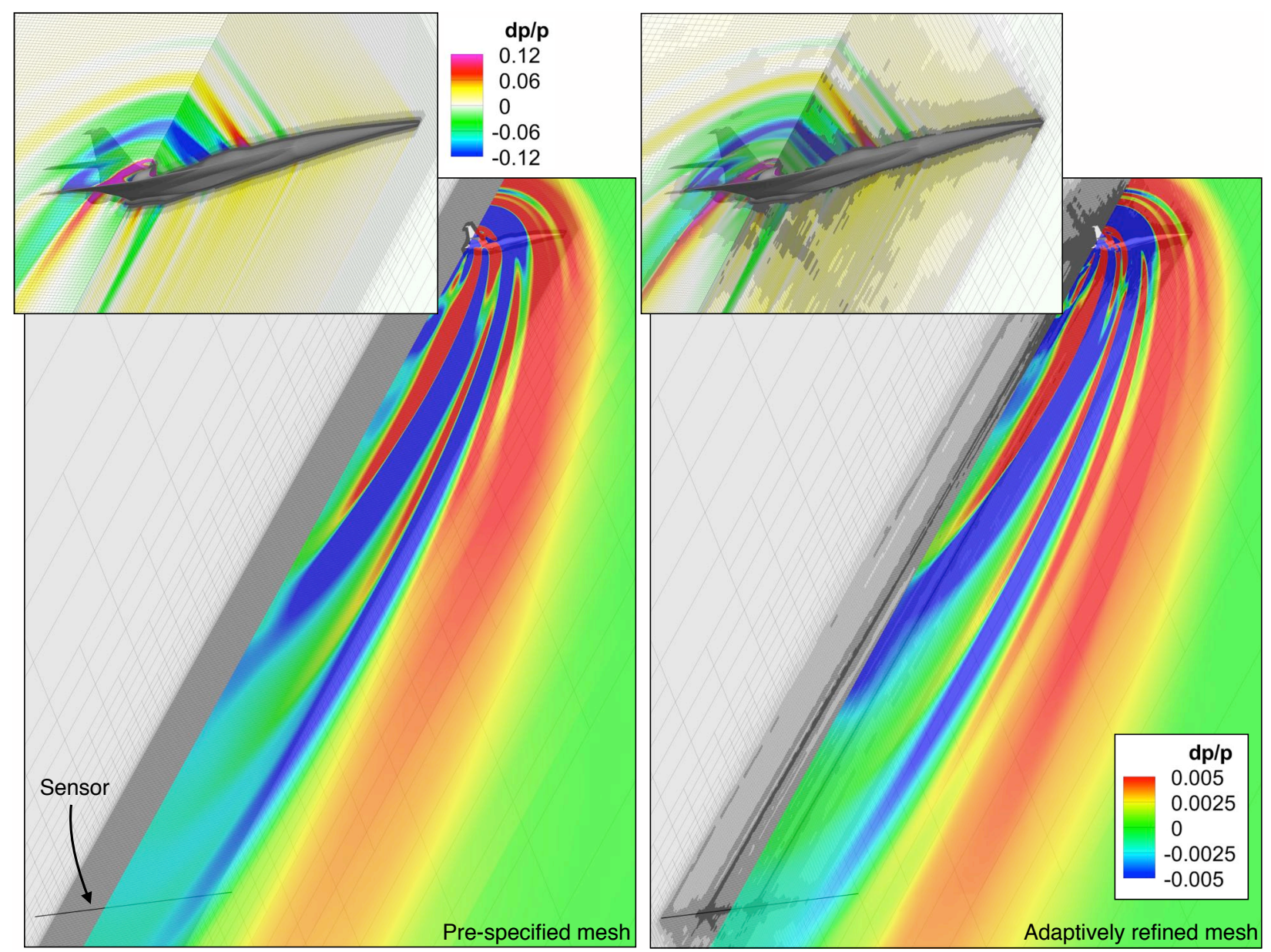

Figure 29. Comparison of design mesh with pre-specified refinement ( 56 million cells), and adaptive refinement (100 million cells). Inset regions show pressure change and near-body refinement on symmetry and $z=60 \mathrm{ft}$ planes, while large panels show nearfield pressures sampled on the $z=70 \mathrm{ft}$ plane, along with mesh refinement at the symmetry plane; note distinct pressure mapping in each case. $M_{\infty}=1.6, \alpha=3.375^{\circ}$ 
To address this discrepancy, the pre-specified mesh was revised based on observations of the adapted mesh structure. Additional refinement focused on decreasing dissipation between the nozzle region and nearfield sensor yielded an 85 million cell mesh able to capture the aft shock. The nearfield pressure for the design developed within this mesh shows that the shock has been eliminated, as plotted in Fig. 30(a). Loudness of the ground signature, shown in Fig. 31(b), is reduced to 79.1 PLdB.

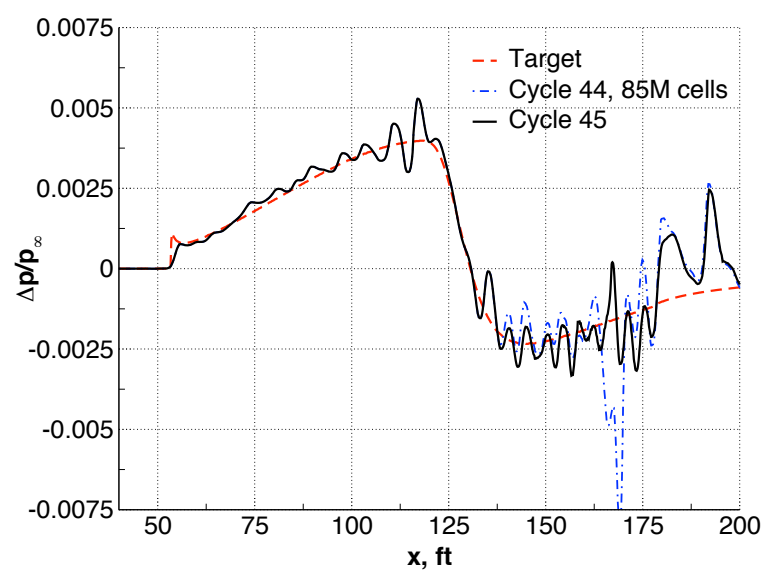

(a) Nearfield pressure signatures sampled at $h / L=3$

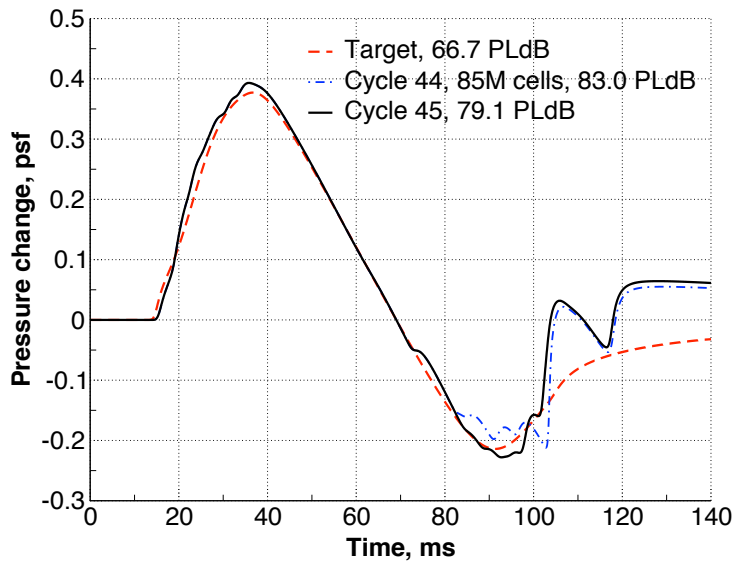

(b) Propagated ground signatures

Figure 30. Nearfield pressure and ground signature propagated from the 51,700 $\mathrm{ft}$ cruise condition for the cycle 45 OML. The cycle 44 solution obtained from the revised, 85 million cell mesh is shown for reference. $M_{\infty}=1.6$

However, evaluating the cycle 45 design using an adaptively refined mesh yields a nearfield pressure with substantial aft differences relative to the pre-specified, 85 million cell solution, as shown in Fig. 31(a). The pre-specified mesh exhibits high-frequency "rattle" over the interval $140 \leq x \leq 170$, and terminates with two strong shocks, neither of which manifest in the adapted mesh. While the loudness change for the ground signatures, shown in Fig. 31(b), is a relatively small 1 PLdB, the aft character of the pressure signals also differs substantially.

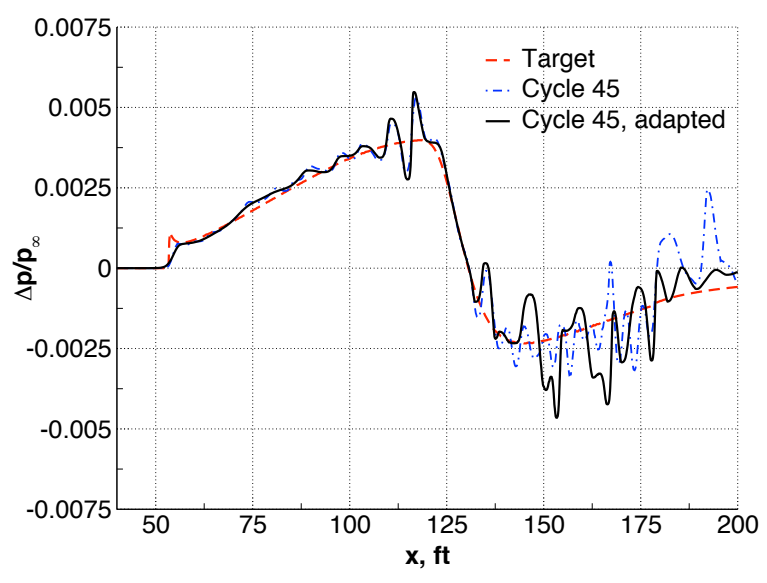

(a) Nearfield pressure signatures sampled at $h / L=3$

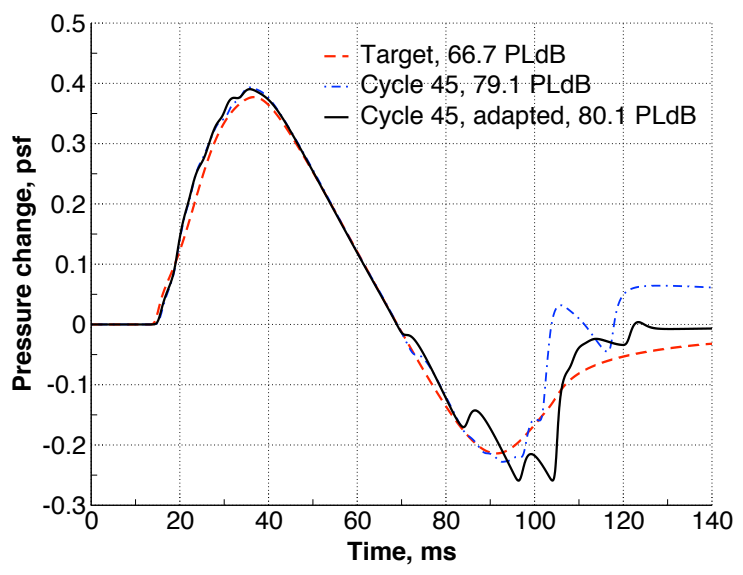

(b) Propagated ground signatures

Figure 31. Nearfield pressure and ground signature propagated from the 51,700 ft cruise condition for the cycle $45 \mathrm{OML}$. The adapted mesh contained 100 million cells. $M_{\infty}=1.6$

While it may be possible to resolve these differences with further revisions to the mesh, consider that at this point, the wallclock time for a function evaluation has almost doubled ${ }^{\mathrm{b}}$ relative to the 60 million cell $^{\circ}$

\footnotetext{
${ }^{\mathrm{b}}$ Roughly 90 minutes on the 256-core system described in footnote a.
} 
design mesh. The required mesh resolution is such that the computational resource outlay within a function evaluation is commensurate with the application of mesh adaptation.

With that in mind, a final design cycle was performed invoking adjoint-driven mesh refinement during shape optimization. The adapted mesh contained roughly 50 million cells at each function evaluation. Symmetry plane cuts of the pre-specified, 85 million cell mesh and adapted, 50 million cell mesh are compared in Fig. 32 for the cycle 45 OML. The respective nearfield signals are overlaid for each mesh. At this refinement

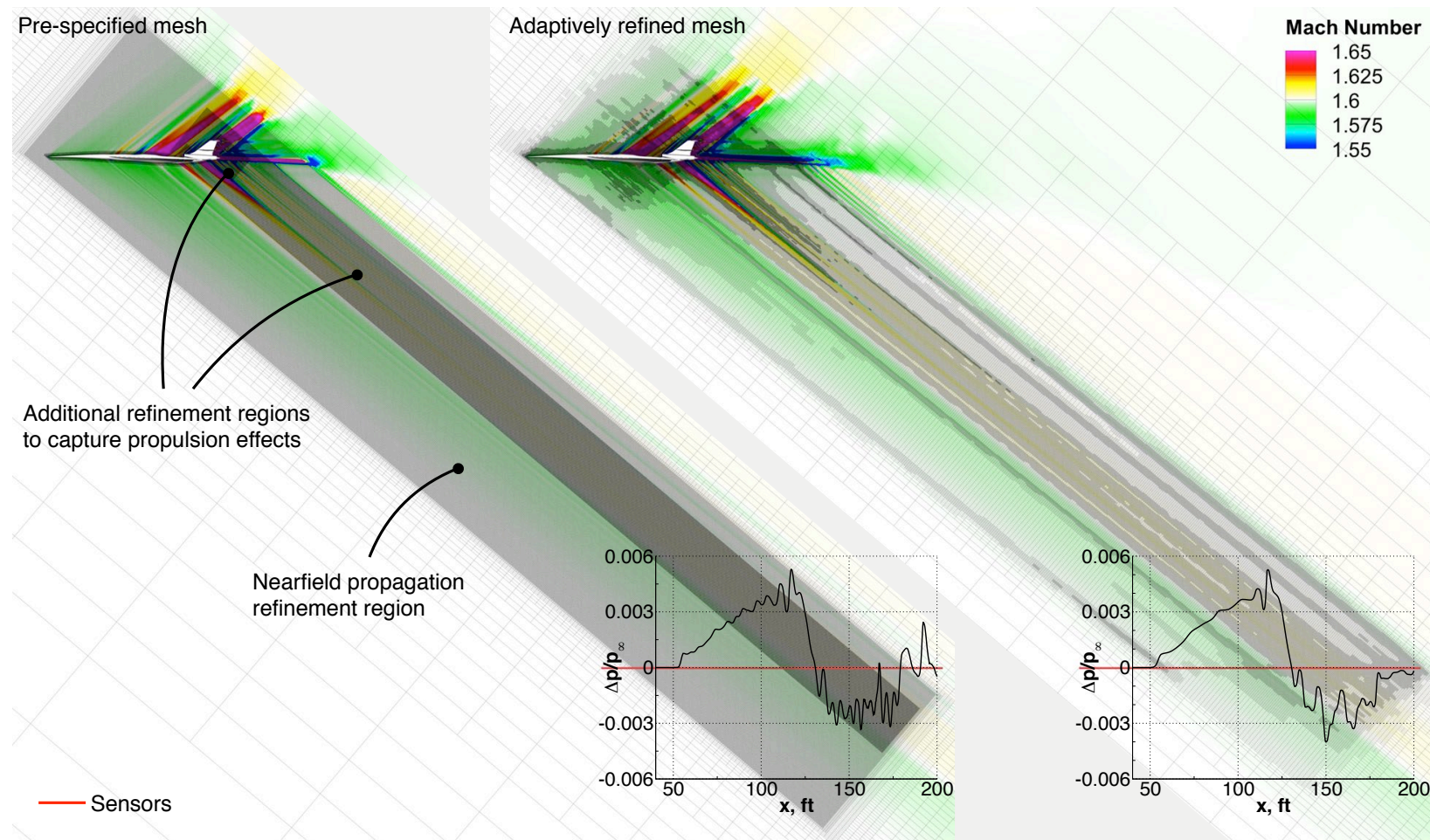

Figure 32. Comparison of 85 million cell pre-specified mesh used during cycle 45 shaping, and 50 million cell adapted mesh used to obtain the cycle 46 OML. Nearfield pressure signals sampled from each mesh are placed at their respective sensor locations. $M_{\infty}=1.6, \alpha=3.375^{\circ}$

level, signal character and shock placement was consistent with a more deeply refined, 100 million cell mesh. Shock amplitudes were noticeably smaller due to dissipation; this was tolerated as a means to reduce iteration times, which were increased roughly $30 \%$ over the 85 million cell design mesh ${ }^{c}$. In the pre-specified mesh, the terminating shocks can be observed to align with the refinement boundaries of the "additional" and "nearfield propagation" regions. This clearly demonstrates how an artifact of the pre-specified mesh structure can give rise to spurious signal features, and strongly supports use of mesh adaptation during this latter stage of OML shaping.

Nearfield pressures for the cycle 45 design are compared with the final, cycle 46 design in Fig. 33(a); both signals were sampled from adapted meshes containing roughly 100 million cells. While the signals remain very similar in overall appearance, the revised design crucially breaks up the large-wavelength oscillations at $x \approx 150 \mathrm{ft}$ and $x \approx 160 \mathrm{ft}$, present in the cycle 45 design, into a series of smaller shocks. Propagated to the ground, the resulting ground signature, shown in Fig. 33(b), yields a predicted loudness reduction of almost 4 PLdB, to 76.4 PLdB. At this final cycle, sonic boom performance has been partially recovered relative to the flow-through design; however, trim and aerodynamic performance are compromised, with $x_{\mathrm{cp}}=82.6 \mathrm{ft}$, $C_{L}=0.059$, and inviscid $\mathrm{L} / \mathrm{D}=6.4$.

\section{III.B.4. Lift Deficit}

In the context of sonic boom, the lift deficit that manifests when re-tailoring in the powered state can best be visualized in the under-track-derived $A_{e}$ for powered and unpowered configurations. Figure 34 compares $A_{e}$ curves sampled from the flow-through-optimized (cycle 38) design in both the powered and unpowered

\footnotetext{
${ }^{\mathrm{c}}$ Roughly 2 hours on the 256 -core system described in footnote a.
} 


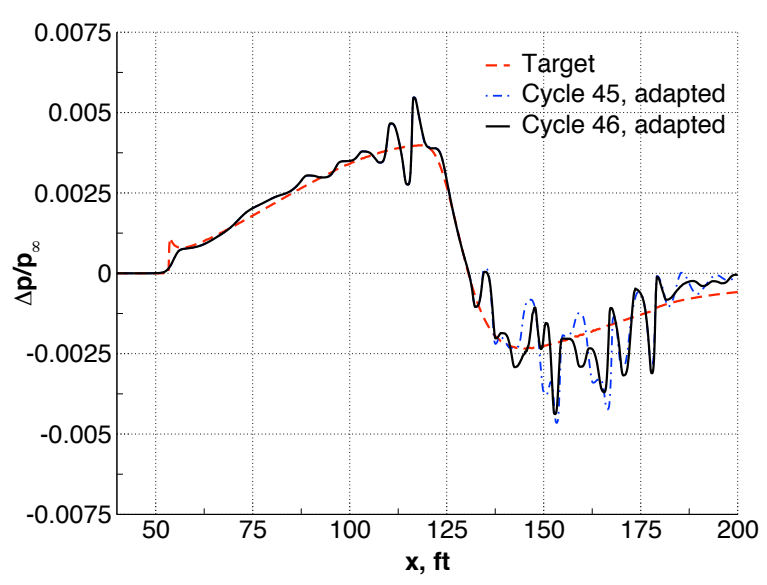

(a) Nearfield pressure signatures sampled at $h / L=3$

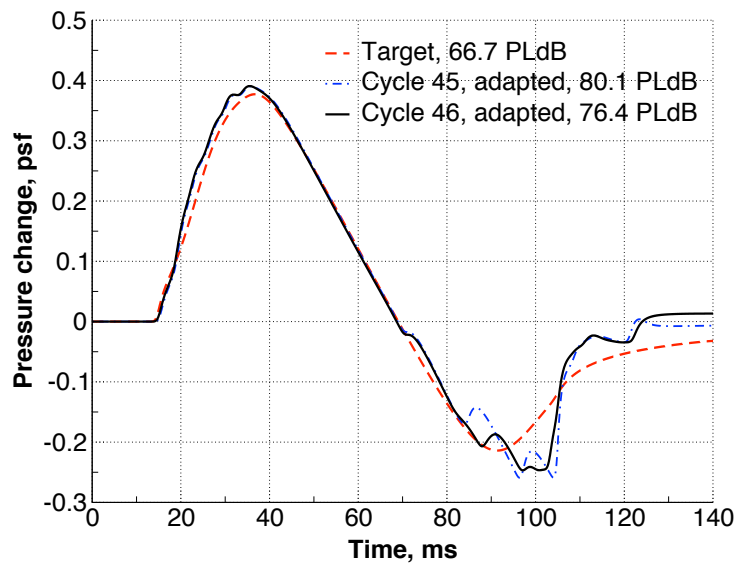

(b) Propagated ground signatures

Figure 33. Nearfield pressure and ground signature propagated from the $51,700 \mathrm{ft}$ cruise condition for the cycle $46 \mathrm{OML}$. Solutions obtained on adaptively refined meshes containing 100 million cells; the cycle 45 solution is shown for reference. $M_{\infty}=1.6$

state, along with the re-tailored, cycle 44 configuration with plug nozzle. Focusing for the moment on the cycle 38 design, the activation of power is shown to result in an area surplus accumulating from the $100 \mathrm{ft}$ effective length mark. As the OML between the two states is identical, and $C_{L}$ is unchanged, the inflow and outflow BCs have altered the flow field in such a way that effective volume has increased. Moving to the cycle 44 curve, representing the powered, plug nozzle design, the $A_{e}$ is seen to compare favorably with that of the flow-through design. When considering that wing and horizontal tail parameters were frozen following flow-through optimization (cycle 38), and that volume changes due to re-shaping with the plug nozzle cannot account for the effective volume loss, the only means by which the optimizer could reduce $A_{e}$ was by reducing the contribution due to lift.

Figures 35(a) and (b) compare $C_{p}$ contours for the flow-through cycle 38 and powered cycle 44 designs, and clearly illustrate this relative loss of lift. Up to the inlet, the upper and lower surface $C_{p}$ contours are almost identical, as would be expected based on the similarity of their nearfield pressures to this point. Starting at the inlet, however, the powered configuration has a larger region of high pressure on the wing upper surface due to the inlet shock. Further downstream, the nacelle lower surface has a larger suction region, though a following high pressure region may balance this somewhat. Finally, while the horizontal tail upper surface contours are similar between the two configurations, the lower surface suction is increased for the powered case. Taken together, these features are consistent with the net reduction in lift.

It is noted that the impact on aerodynamic and boom performance when transitioning to the powered state is configuration dependent, and experience with alternate vehicle layouts has shown that it can be negligible for certain configurations, such as those employ-

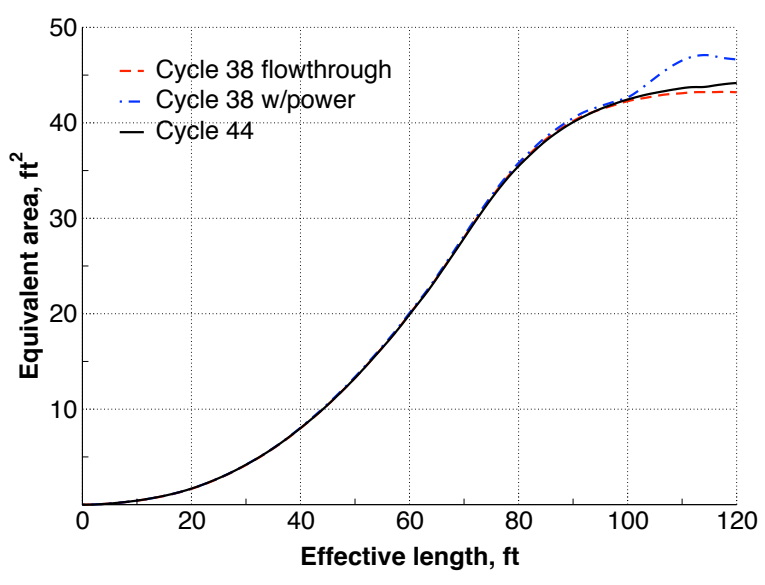

Figure 34. $A_{e}$ curves integrated from the under-track nearfield pressure signals sampled at $h / L=3$ for the flow-through optimum (cycle 38), with and without power, and the re-tailored plug nozzle design (cycle 44). $M_{\infty}=1.6$ ing inlet and nozzle shielding. 


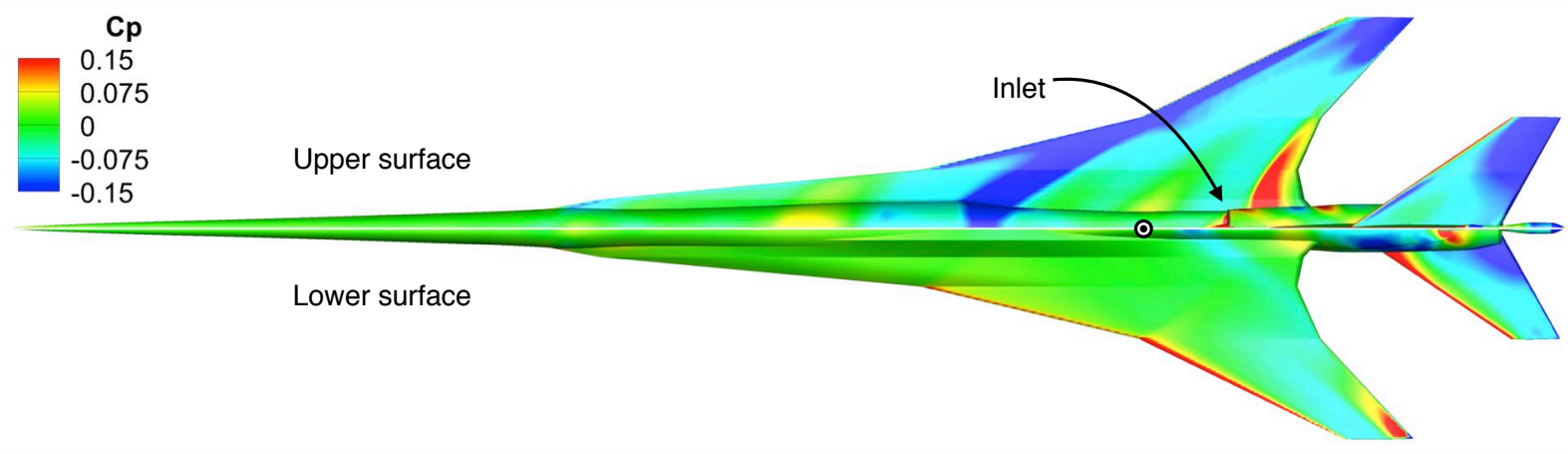

(a) Flow-through optimum (cycle 38), unpowered

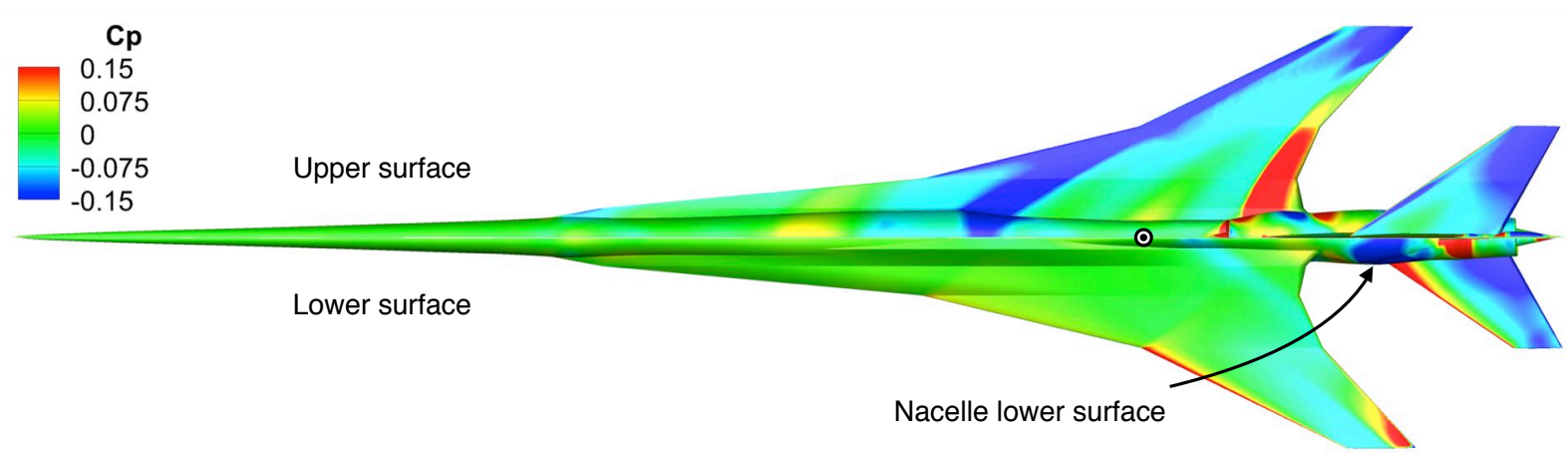

(b) Plug nozzle re-tailored (cycle 44), powered

Figure 35. Comparison of $C_{p}$ contours plotted on the cycle 38 and cycle 44 designs. The $\odot$ symbol indicates the $x$ cp location for trim, nominally at $x=82 \mathrm{ft} . M_{\infty}=1.6, \alpha=3.375^{\circ}$

\section{Conclusions and Future Work}

This work successfully demonstrates the application of powerful, adjoint-based techniques to the undertrack, low boom shape optimization of a conceptual, demonstrator-class vehicle. Lift and trim constraints were implicitly imposed using a technique incorporating both during objective formulation. To ensure development of a realistic OML, geometric constraints were developed from carefully constructed models of large internal components, and imposed on the parametric model during shape optimization. Periodic checks of fuel tankage and CG were also performed to ensure adequate fuel was carried, and could be placed in a way that did not invalidate trim.

Gradient-driven shape optimization of the vehicle was performed in both flow-through and powered states, with flow solutions computed using a nonlinear CFD solver capable of accurately capturing the supersonic shock system about the aircraft. Refinement, enrichment, and extension of the model parameterization was guided by observation of the adjoint field at each design cycle. The flexible modeling approach allowed subtle changes such as re-distribution of defining stations, to more visible changes such as addition of the tail pod. During the early design phase, cycle throughput was increased by the use of carefully constructed, compact nearfield grids. Subsequently, the vehicle OML was re-tailored using expanded, pre-specified grids more appropriate to the propagation theory used. Final OML refinement invoked adjoint-driven, adaptive mesh refinement as part of the shaping process, enabling capture of flow features missed by hand-crafted grids.

Shape optimization for the flow-through configuration demonstrated an almost 10 PLdB reduction relative to the feasible baseline. A particular point of interest was the substantial wing geometry change pursued by the optimizer - and subsequent improvement in both boom and aerodynamic performance - when incorporating the revised main landing gear geometry. When initially transitioning to the powered state, this improvement was largely erased, and analysis in the $A_{e}$ space indicated that the current optimization target does not account for the flow field effect of active power BCs. As a result, any improvement in sonic boom performance required a trade-off in lift, resulting in a $9 \%$ deficit in the final powered design. That said, OML re-tailoring, coupled with the substitution of a plug nozzle geometry in place of the conventional C-D 
nozzle, produced a powered, Mach 1.6 design with sonic boom loudness of 76.4 PLdB.

Future work will initially focus on revising the target to consider powered effects, and subsequently re-tailoring the design to recover trim and lift performance. The current design was performed for a single cruise point; multi-point design is an area of active research, both from a full-carpet, as well as full-mission perspective. Finally, uncertainty quantification, particularly in relation to the interaction of vehicle OML, cruise state, and atmospheric conditions will be explored in future studies.

\section{Acknowledgements}

This work was supported by TEAMS2 contract NNL12AA09C, and by the NASA Fundamental Aeronautics Program Commercial Supersonic Technology Project. The authors gratefully acknowledge Michael Aftosmis (NASA Ames Research Center) and Marian Nemec (Science \& Technology Corp.) for assistance with applying the Cart3D adapted mesh shape design capability. The authors also wish to thank Karl Geiselhart (NASA Langley Research Center) for guidance and data provided during propulsion integration, and Sriram Rallabhandi (National Institute of Aerospace) for sBOOM support, and many helpful discussions.

\section{References}

${ }^{1}$ George, A. R. and Seebass, R., "Sonic Boom Minimization Including Both Front and Rear Shocks," AIAA Journal, Vol. 9, No. 10, October 1971, pp. 2091-2093.

${ }^{2}$ Seebass, R. and George, A. R., "Sonic-Boom Minimization," Journal of the Acoustical Society of America, Vol. 51, No. 2C, January 1972, pp. 686-694.

${ }^{3}$ Darden, C. M., "Sonic-Boom Minimization with Nose-Bluntness Relaxation," Technical Paper TP-1348, NASA, NASA Langley Research Center, Hampton, VA, January 1979.

${ }^{4}$ Farhat, C., Maute, K., Argrow, B., and Nikbay, M., "Shape Optimization Methodology for Reducing the Sonic Boom Initial Pressure Rise," AIAA Journal, Vol. 45, No. 5, May 2007, pp. 1007-1018.

${ }^{5}$ Chan, M. K., Supersonic Aircraft Optimization for Minimizing Drag and Sonic Boom, Ph.D. dissertation, Stanford University, Stanford, CA, August 2003.

${ }^{6}$ Alonso, J. J., Kroo, I. M., and Jameson, A., "Advanced Algorithms for Design and Optimization of Quiet Supersonic Platforms," 40th AIAA Aerospace Sciences Meeting and Exhibit, No. 2002-0144, AIAA, Reno, NV, January 2002.

${ }^{7}$ Choi, S., Alonso, J. J., Kroo, I. M., and Wintzer, M., "Multifidelity Design Optimization of Low-Boom Supersonic Jets," Journal of Aircraft, Vol. 45, No. 1, January-February 2008, pp. 106-118.

${ }^{8}$ Alauzet, F. and Loseille, A., "High-Order Sonic Boom Modeling Based on Adaptive Methods," Journal of Computational Physics, Vol. 229, No. 3, 2010, pp. 561-593.

${ }^{9}$ Park, M. A. and Darmofal, D. L., "Output-Adaptive Tetrahedral Cut-Cell Validation for Sonic Boom Prediction," 26th AIAA Applied Aerodynamics Conference, No. 2008-6594, AIAA, Honolulu, HI, August 2008.

${ }^{10}$ Nemec, M., Aftosmis, M. J., and Wintzer, M., "Adjoint-Based Adaptive Mesh Refinement for Complex Geometries," 46th AIAA Aerospace Sciences Meeting, No. 2008-0725, AIAA, Reno, NV, January 2008.

${ }^{11}$ Wintzer, M., Nemec, M., and Aftosmis, M. J., "Adjoint-Based Adaptive Mesh Refinement for Sonic Boom Prediction," 26th AIAA Applied Aerodynamics Conference, No. 2008-6593, AIAA, Honolulu, HI, August 2008.

${ }^{12}$ Nadarajah, S. K., Kim, S., Jameson, A., and Alonso, J. J., "Sonic Boom Reduction using an Adjoint Method for Supersonic Transport Aircraft Configurations," IUTAM Symposium Transsonicum IV, edited by H. Sobieczky, Vol. 73 of Fluid Mechanics and Its Applications, DLR, Gottingen, Germany, September 2002.

${ }^{13}$ Aftosmis, M. J., Nemec, M., and Cliff, S. E., "Adjoint-Based Low-Boom Design with Cart3D," 29th AIAA Applied Aerodynamics Conference, No. AIAA 2011-3500, AIAA, Honolulu, HI, June 2011.

${ }^{14}$ Wintzer, M. and Kroo, I. M., "Optimization and Adjoint-Based CFD for the Conceptual Design of Low Sonic Boom Aircraft," 50th AIAA Aerospace Sciences Meeting, No. AIAA-2012-0963, AIAA, Nashville, TN, January 2012.

${ }^{15}$ Palacios, F., Alonso, J. J., Colonno, M., Hicken, J., and Lukaczyk, T., "Adjoint-Based Method for Supersonic Aircraft Design Using Equivalent Area Distributions," 50th AIAA Aerospace Sciences Meeting, No. AIAA 2012-0269, AIAA, Nashville, TN, January 2012.

${ }^{16}$ Morgenstern, J. M., Buonanno, M., and Nordstrud, N., "N+2 Low Boom Wind Tunnel Model Design and Validation," 30th AIAA Applied Aerodynamics Conference, No. AIAA 2012-3217, AIAA, New Orleans, LA, June 25-28 2012.

${ }^{17}$ Magee, T. E., Shaw, S. G., and Fugal, S. R., "Experimental Validations of a Low-Boom Aircraft Design," $51 s t$ AIAA Aerospace Sciences Meeting, No. AIAA 2013-0646, AIAA, Grapevine, TX, January 7-10 2013.

${ }^{18}$ Wintzer, M., Optimization and Adjoint-Based CFD for the Conceptual Design of Low Sonic Boom Aircraft, Ph.D. dissertation, Stanford University, Stanford, CA, August 2012.

${ }^{19}$ Conners, T. R. and Howe, D. C., "Supersonic Inlet Shaping for Dramatic Reductions in Drag and Sonic Boom Strength," 44th AIAA Aerospace Sciences Meeting and Exhibit, No. 2006-0030, AIAA, Reno, NV, January 9-12 2006.

${ }^{20}$ Slater, J. W., "Methodology for the Design of Streamline-Traced External-Compression Supersonic Inlets," 50th AIAA Joint Propulsion Conference, No. 2014-3593, AIAA, Cleveland, OH, July 28-30 2014.

${ }^{21}$ Benson, T. J., "An Interactive Method of Characteristics Java Applet to Design and Analyze Supersonic Aircraft Nozzles," 50th AIAA Joint Propulsion Conference, No. 2014-4011, AIAA, Cleveland, OH, July 28-30 2014. 
${ }^{22}$ Heath, C. M., Gray, J. S., Park, M. A., Nielsen, E. J., and Carlson, J.-R., "Aerodynamic Shape Optimization of a Dual-Stream Supersonic Plug Nozzle," 53rd AIAA Aerospace Sciences Meeting, No. 2015-1047, AIAA, Kissimmee, FL, January 5-9 2015.

${ }^{23}$ Graham, D. H., Dahlin, J. H., Meredith, K. B., and Vadnais, J., "Aerodynamic Design of Shaped Sonic Boom Demonstration Aircraft," 43rd AIAA Aerospace Sciences Meeting and Exhibit, No. 2005-0008, AIAA, Reno, NV, January 2005.

${ }^{24}$ Ordaz, I., Wintzer, M., and Rallabhandi, S. K., "Full Carpet Design of a Low-Boom Demonstrator Concept," 33rd AIAA Applied Aerodynamics Conference, AIAA, Dallas, TX, June 22-26 2015.

${ }^{25}$ Ordaz, I., Geiselhart, K. A., and Fenbert, J. W., "Conceptual Design of Low-Boom Aircraft with Flight Trim Requirement," 32nd AIAA Applied Aerodynamics Conference, No. 2014-2141, NASA Langley Research Center, Atlanta, GA, June $16-202014$.

${ }^{26}$ Wintzer, M., Castner, R., and Geiselhart, K. A., "Airframe-Nozzle-Plume Interactions in the Context of Low Sonic Boom Design," 53rd AIAA Aerospace Sciences Meeting, Kissimmee, FL, January 5-9 2015.

${ }^{27}$ Anderson, G. R. and Aftosmis, M. J., "Adaptive Shape Control for Aerodnyamic Design," 56th AIAA/ASCE/AHS/ASC Structures, Structural Dynamics, and Materials Conference, No. 2015-0398, AIAA, Kissimmee, FL, January 5-9 2015.

${ }^{28}$ Rallabhandi, S. K., "Advanced Sonic Boom Prediction Using the Augmented Burgers Equation," Journal of Aircraft, Vol. 48, No. 4, July-August 2011, pp. 1245-1253.

${ }^{29}$ Committee S1, Acoustics, "Method for Calculation of the Absorption of Sound by the Atmosphere, Annex C," ANSI Standard S1.26-1995, American National Standards Institute, New York, NY, September 1995.

${ }^{30}$ Stevens, S. S., "Perceived Level of Noise by Mark VII and Decibels (E)," The Journal of the Acoustical Society of America, Vol. 51, No. 2B, February 1972, pp. 575-601.

${ }^{31}$ Shepherd, K. P. and Sullivan, B. M., "A Loudness Calculation Procedure Applied to Shaped Sonic Booms," Technical Paper TP 3134, NASA Langley Research Center, Hampton, VA, November 1991.

${ }^{32}$ Aftosmis, M. J., Berger, M. J., and Melton, J. E., "Robust and Efficient Cartesian Mesh Generation for Component-Based Geometry," AIAA Journal, Vol. 36, No. 6, June 1998, pp. 952-960.

${ }^{33}$ Nemec, M. and Aftosmis, M. J., "Adjoint sensitivity computations for an embedded-boundary Cartesian mesh method," Journal of Computational Physics, Vol. 227, 2008, pp. 2724-2742.

${ }^{34}$ Gill, P. E., Murray, W., and Saunders, M. A., User's Guide for SNOPT Version 7: Software for Large-Scale Nonlinear Programming, Department of Mathematics, University of California, San Diego, La Jolla, CA, February 2006.

${ }^{35}$ Aftosmis, M. J., "Solution Adaptive Cartesian Grid Methods for Aerodynamic Flows with Complex Geometries," Lecture Series 1997-02, von Karman Institute for Fluid Dynamics, Chaussée de Waterloo 72, B-1640 Rhode-Saint-Genèse, Belgium, March 3-7 1997.

${ }^{36}$ Nemec, M. and Aftosmis, M. J., "Output Error Estimates and Mesh Refinement in Aerodynamic Shape Optimization," 51st AIAA Aerospace Sciences Meeting, No. 2013-0865, AIAA, Grapevine, TX, January 7-10 2013.

${ }^{37}$ Kulfan, B. M., "Universal Parametric Geometry Representation Method," Journal of Aircraft, Vol. 45, No. 1, JanuaryFebruary 2008, pp. 142-158.

${ }^{38}$ Hahn, A. S., "Vehicle Sketch Pad: Parametric Geometry for Conceptual Aircraft Design," 48th AIAA Aerospace Sciences Meeting, No. 2010-0657, AIAA, Orlando, FL, January 4-7 2010.

${ }^{39}$ Rallabhandi, S. K., "Application of Adjoint Methodology in Various Aspects of Sonic Boom Design," 32nd AIAA Applied Aerodynamics Conference, No. 2014-2271, AIAA, Atlanta, GA, June 16-20 2014.

${ }^{40}$ Sommer, S. C. and Short, B. J., "Free-Flight Measurements of Turbulent-Boundary-Layer Skin Friction in the Presence of Severe aerodynamic Heating at Mach Numbers from 2.8 to 7.0," Technical Note 3391, National Advisory Committee for Aeronautics, Moffett Field, CA, March 1955.

${ }^{41}$ Lytle, J., Follen, G., Naiman, C., Evans, A., Veres, J., Owen, K., and Lopez, I., "Numerical Propulsion System Simulation (NPSS) 1999 Industry Review," Technical Memorandum 2000-209795, NASA Glenn Research Center, Cleveland, OH, August 2000 . 